\title{
Replication of an Experiment on Linguistic Tool Support for Consolidation of Requirements from Multiple Sources
}

\author{
Krzysztof Wnuk · Martin Höst · Björn Regnell
}

Received: date / Accepted: date

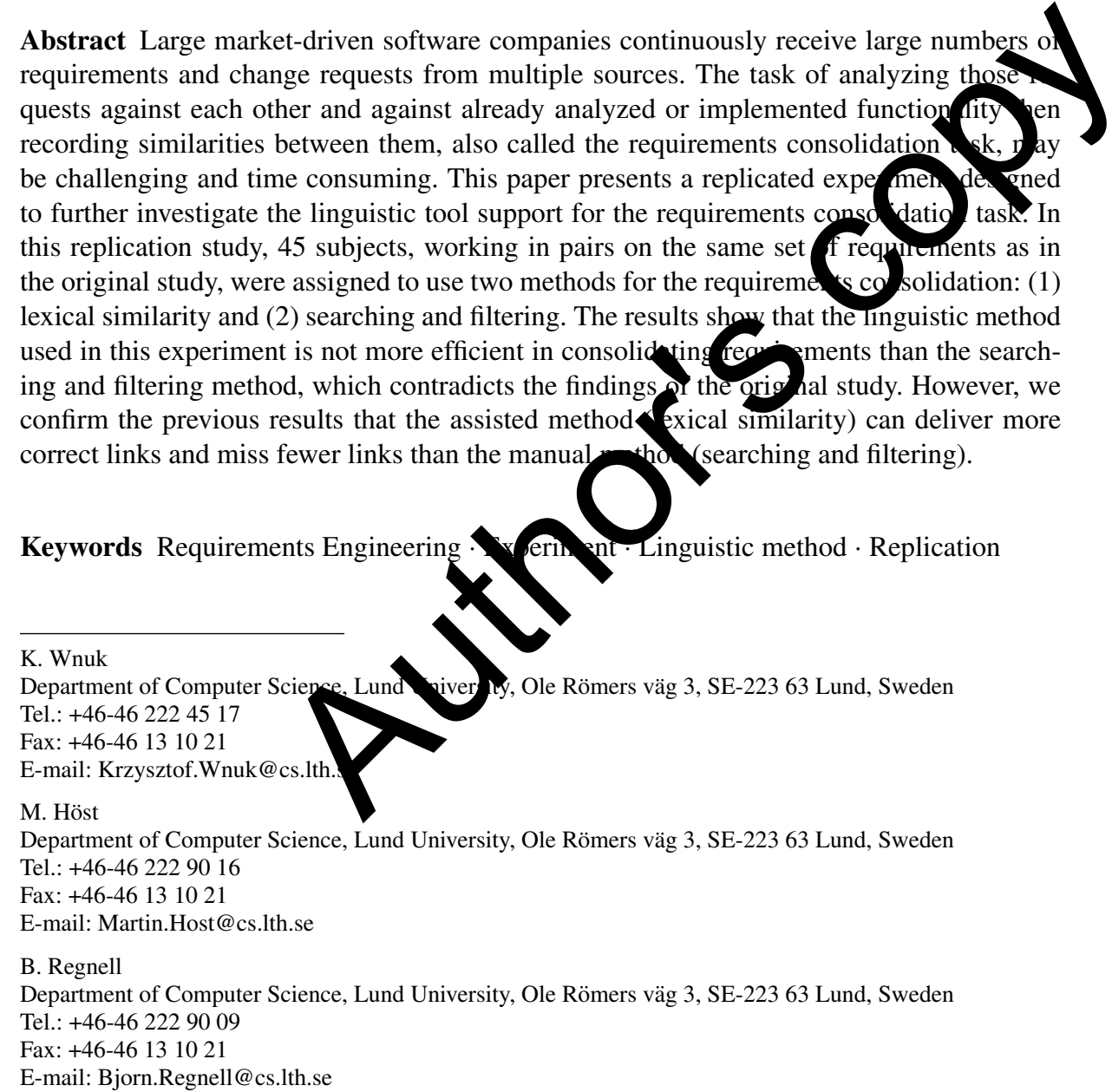




\section{Introduction}

Requirements engineering in a market-driven context can be characterized by continuous elicitation, time-to-market constraints, and strong market competition (Natt och Dag 2006a, Regnell and Brinkkemper 2005). In this context, requirements are continuously arriving from multiple sources, throughout the development process (Regnell et al 1998). When the company is growing and expanding, more products are created which result in a more complex variability structure, and more effort is needed to handle product customizations, for example by utilizing the Software Product Line (SPL) concept (Pohl et al 2005). This constant flow of requirements needs to be analyzed from the perspective of new market opportunities and technical compliance. In a case when a company is large and develops complex software solutions, the quantity of information to constantly analyze and assess may severely impede the analytical capacity of requirements engineers and managers (Gorschek et al 2007, Leuser 2009). Providing a method that can assist in analyzing large numbers of natural language requirements for the purpose of finding and recording similarities between them can significantly reduce time needed to perform the task (Cleland-Huang et al 2007), help to miss fewer requirements links (Natt och Dag et al 2006) and increase the accuracy of the task.

The process of analyzing incoming requirements from customers or customer representatives (also called proxy-customers) against requirements already present in the requiro ments repository can be called requirements consolidation. This process included athe ing
incoming documents, finding similarities, and merging or linking similar desarip. ins a consolidated single description that covers all analyzed aspects. This pro ess o p a be a part of the broader impact analysis task. The core of the requirementom olid tion process is finding the similarities between requirements and recording em b making links between them (Natt och Dag et al 2006). However, the number of ssi le links grows exponentially with the increase of the number of requiremen analyze, which may result in overwhelming the company's management and aly (Leuser 2009). As a remedy to this problem, Natt och Dag et al. (2006) dey oped and evaluated a method for requirements consolidation that utilizes linguistic tech ques and provides a list of requirements that are the most similar to the currently revequirement. The evaluation of this method showed that using the method can si (ifica ly improve the performance of the consolidation process as well as the numer linked requirements, and that it can help to miss fewer requirements links (Na gch o et al 2006). However, the unsupported method used in the original experimek was nited to a simple search functionality, while most currently available requiremen man tools offer more advanced filtering and searching techniques.

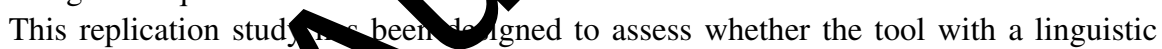
analysis of the similarity bu we nn wirements can still perform better than currently available commercial requiremen management tools in the task of requirements consolidation. A replicated experiment has chosen due to its falsifiable nature. Replications provide a possibility to evaluate whether the output parameters of a system remain stable if one or more input parameters are systematically changed.

In this experiment, two subject groups, working in pairs, were asked to consolidate two requirements sets by finding and linking requirements that address the same underlying functionality. This replication reuses the original procedures in terms of the study design, experimental steps and the two requirement sets. The changes to the original experiment are: (1) using another set of subjects which were asked to work in pairs due to unexpected housing issues and (2) changing one of the treatments. Due to a limited number of available 
computers in the laboratory room, the subjects were asked to work in pairs on the assignment. Given this context, this replication study can be classified according to Shull et al. (2008) as a dependent replication. However the classification provided by Shull does not define if a replication where both the population and one of the methods used to test the hypotheses is changed can also be categorized as an exact replication (Shull et al. (2008) only mention changing either the population or the artifact on which the technique is applied). According to the classification by Basili et al. (1999), this replication type is the one that varies the research hypotheses. The unchanged object in this replication study, also called the assisted method, is a research prototype tool, called ReqSimile (Natt och Dag 2006b), that utilizes linguistic analysis to assist in the task of finding similar requirements. The second object, which was changed compared with the original experiment, is called the manual method, and it utilizes searching and filtering functionalities implemented in a tool called Telelogic Doors (IBM 2010a). ${ }^{1}$

The objectives of the study are twofold: firstly to assess if a significant differences between the two methods tested in the original experiment can be confirmed in a replicated experiment setup and secondly to compare the results for the same methods between the two experimental sessions. The objectives are refined to two main research questions in Section 4.

The paper is structured as follows: Section 2 provides industrial problem description. Section 3 provides related work. Section 4 describes the experimental design. Section 5 ex plains experiment execution procedures. Section 6 describes the experiment resul analy is. Section 7 provides an interpretation of results. Section 8 concludes the paper

\section{Industrial Problem Description}

New requirements and changes to existing requirements are in sitome situations at all stages of the system development process (Kotonya and Somme vill-1999). The two principal requirements management activities that address this phe omena are: (1) change control and (2) change impact assessment. The change contromens that, if a change is accepted, its impact on design and implementation artifa s wh be addressed. The change impact assessment warrants that proposed changes baye $\mathrm{kno} / \mathrm{n}$ impact on the requirements and software system (Kotonya and Sommervi 1990. A company that is operating in a marketdriven mode should continuously mon th market situation by checking competitors latest achievements, researching mark ne and collecting all possible feedback from the market in a chase for achieving or man vinng the competitive advantage within its operational business. This purs after on mal market window, together with other reasons, creates a constant flow of ew irements and ideas throughout the entire software product lifetime (Karlsson et al 12). As a result, the requirements process for market-driven contexts needs to be enriched with procedures to capture and analyze this constant flow of requirements (Higgins et al 2003).

As pointed out by practitioners from large companies (Berenbach et al 2009), when development projects grow in complexity and new products are released to the market with many features, the importance of good practices in requirements management grows. In the

\footnotetext{
1 The Telelogic DOORS tool has recently changed its vendor and its name to Rational DOORS. However, since the Telelogic Doors version 8.3 was used in this experiment, we will refer to this tool throughout this paper as Telelogic Doors. Both methods were compared for the task of requirements consolidation meaning that comparing the two tools in general is outside of the scope of this paper.
} 
case when a company is large and operates globally, the diversity of customers and the complexity of software products can make the list of sources of new requirements and change requests extensively long, including: customers and proxy-customers (marketing, customer representatives and key account managers), suppliers, portfolio planners and product managers. The company's requirements analysts should, in this, case analyze all incoming requirements and change requests in order to find an optimal set of requirements that will address the needs of as many customers as possible. In this context, the concept of Software Product Lines (SPL) (Pohl et al 2005) is often used to increase the reuse of common components while providing necessary diversity of similar products, requested by various customers.

Change management in a Software Product Lines context can be particularly challenging, for example, because of the extensive and often exhaustive variability analysis that has to be performed while analyzing the impact of a change. Moreover, the requirements analyst has to consider if a certain new requirement or request has already been analyzed and what was the result of this analysis. One of the methods to assist with the analysis of incoming requirements versus those already present in the requirements database is to find and record similarities, making traceability links. In the industrial case example, provided by the original experiment, the experts became frustrated during the analysis because the had to identify compliance to the same or very similar requirements multiple times. Large parts of the new versions of requirements request documents, arriving from the samecus tomer are typically the same as previous versions. Furthermore, the same and ve sin, $\mathrm{Yar}$
requirements can appear in the request from different customers (Natt och Dacet 20). Providing an automatic or semi-automatic method of analyzing similarity be ween nc ning requirements could significantly decrease the amount of time needed to $\mathrm{cm} t / \mathrm{s}$ task.

The process of finding and recording similarities between software levelo $p$ ment artifacts is a part of the requirements traceability activity, which has been wh cognized as a useful method for recording relations and dependencies betw software project artifacts for the purposes of change and impact analysis tasks an 1 1995, Wiegers 2003, Antoniol et al 2002, Jarke 1998, Gotel and Finkelstein 194). The ore of the requirements traceability task is to find and record the dependencies etween the traced elements, which are assumed to be exhibited by their lexical similar a ch Dag 2006a). The importance of requirement traceability is significant; the U.S Depa ment of Defense invested in 2001 about 4 percent of its total IT budget on ac lid (Ramesh and Jarke 2001). Other large companies, have also stressed perta of implementing traceability in their industry projects (Samarasinghe et al 09, renbach et al 2009, Konrad and Gall 2008, Panis 2010, Leuser 2009).

However, despite recognitio f its sportance, implementing a successful traceability in practice is challenging $\mathrm{g}$ et al 2002). The task of finding relationships between the elements and est bli Altrotraces between them is a "mind numbing" (Hayes et al 2003), error prone and time o nsuming activity. Moreover, maintaining a traceability scheme is difficult because the artifac being traced continue to change and evolve as the system is developed and extended (Zowghi and Offen 1997, Strens and Sugden 1996). Furthermore, as pointed out by Leuser (2009), current traceability approaches used in practice are cumbersome and very time consuming, mainly because they are almost completely manual. The size of requirements specifications in large industrial projects may reach thousands of requirements (Leuser 2009, Konrad and Gall 2008). To tackle these issues, several researchers proposed using Information Retrieval (IR) methods such as the Vector Space Model (VSM), also used in this experiment, (Antoniol et al 2002, Cleland-Huang et al 2007, Natt och Dag et al 2004), the Probabilistic Network Model (Cleland-Huang et al 2005; 2010), and Latent 
Semantic Indexing (LSI) (Lucia et al 2007, Hayes et al 2006, Lormans and Van Deursen 2006, Marcus and Maletic 2003) for semi-automatic recovery of traceability links.

\section{Related Work}

Replications play an important role in software engineering by allowing us to build knowledge about which results or observations hold under which conditions (Shull et al 2008). Unfortunately, replications in software engineering are still rarely reported (Ivarsson and Gorschek 2009). A recent survey of controlled experiments in software engineering revealed that replications are still neglected by empirical researchers, only $18 \%$ of the surveyed experiments are reported as replications (Sjøberg et al 2005). Moreover only 3.9\% of analyzed controlled experiments can be categorized according to the IEEE taxonomy as requirements/specification related (Sjøberg et al 2005, IEEE 2010).

The awareness of new possibilities that Natural Language Processing (NLP) can bring to requirements engineering has been present from the beginning of the requirements engineering discipline, when Rolland et al. (1992) discussed the natural language approach for requirements engineering. Shortly after, Ryan (1993) warned that although natural lan guage processing provides a variety of sophisticated techniques in the requirements engineering field, they can only support sub-activities of requirements engineering and that tho process of using natural language processing techniques has to be guided by pra tition rs.
The possibilities mentioned by Ryan (1993) and Rolland et al. (1992) have later een explored by a number of research studies and publications, where application of va ou VLP techniques in supporting requirements management activities were evalund scussed. Among those that include some kind of empirical evaluations, the ast meiority of natural language process tools are used to examine the quality of requirem secifications in terms of, for example, the number of ambiguities (Fantechi 2003) by assigning ambiguity scores to sentences depending on the degree of nnt a d semantic uncertainty (Macias and Pulman 1995), or detecting ambiguities b applyion inspection technique (Kamsties et al 2001). Furthermore, Rupp et al. (2006 produced logical forms associated with parsed sentences to detect ambiguities. Amor or yality attributes of requirements artifacts that natural language processing attemp to a alyze and improve, Fabbrini et al. (2001) proposed a tool that assesses un ars consistency, testability, and correctness of requirements documents. Providn mea rements that can be used to assess the quality of a requirements specification cun is the aim of the ARM tool proposed by Wilson et al. (1997). Mich et al. (20 roted on an experiment designed to assess the extent to which an NLP tool imp ves t quality of conceptual models. Finally, Gervasi et al. (2000) used natural la pro ing techniques to perform a lightweight validation (low computational and hu ary of natural language requirements.

Apart from the quality o gluation and assurance tasks, NLP techniques have also been applied to the task of extract $\mathrm{g}$ abstractions from textual documents (Aguilera and Berry 1991, Goldin and Berry 1997) and helping combining crucial requirements from a range of documents that include standards, interview transcripts, and legal documents (Sawyer et al 2002). Sawyer at al. (2005) have also reported how corpus-based statistical language engineering techniques are capable of providing support for early phase requirements engineering. Rayson et al. (2001) reported experiences from a project where probabilistic NLP techniques were used to identify and analyze domain abstractions. Their results were further supported by a later study by Sawyer et al. (2004), where ontology charts of key entities were produced using collocation analysis. The continued interest in this issue has been reported 
by Gacitua et al. (2010) who proposed a new technique for the identification of single- and multi-word abstractions named Relevance driven Abstraction Identification (RAI). Finally, Gervasi et al. (1999) used lexical features of the requirements to cluster them according to specific criteria, thus obtaining several versions of the requirements document.

The ReqSimile tool evaluated in this paper uses a correlation to measure lexical similarity and thus rank candidate requirements for linking, presenting to the user the "top" subset of those requirements. The linguistic method, also called the cosine measure, uses a vectorspace representation of requirements where each requirement is represented using a vector of terms with the respective number of occurrences (Natt och Dag et al 2004, Manning and Schütze 2002). Each term can be seen as a dimension in an N-dimensional space while a whole requirement can be represented as a point in the N-dimensional space. Similar requirements will be represented in this space as $m$ points closely clustered. From the matrix, which shows how many times a term appears in each requirement, the information may be derived about how many terms the two requirements have in common. The very similar requirements will result in closely clustered points in this vector space (Manning and Schütze 2002). In the evaluated method (Natt och Dag 2006a) a frequency of terms has been used, instead of counting the occurrences. The cosine correlation measure is often chosen in text retrieval applications for the purpose of finding similar requirements, as it does not depen on the relative size of the input (Manning and Schütze 2002).

$$
\sigma(f, g)=\frac{\sum_{t} w_{f}(t) * w_{g}(t)}{\sqrt{\sum_{t} w_{f}(t)^{2} * \sum_{t} w_{g}(t)^{2}}}
$$

The measure in equation 1 is used for calculating the degree of simi -itv where $f$ and $g$ are two requirements, $t$ ranges over terms, and $w(t)$ denotes theight of term $t$. The term weight is typically a function of the term frequency, sin wh te umber of times a word occurs is relevant, its relevance decreases as the numbergets larod (Manning and Schütze 2002). However, there is a challenge in the way stemm rules are used in this method. For example, the stemming rules do not reduce the ver a rization and the noun container to the same stem. From a semantic point of vie this perfectly correct, but as the two terms concern the same domain concepthe nould be utilized to increase the similarity measure. The realization of the ector pace Model used in this paper does not support this association. Another pote. al $\mathrm{p}$ blem has to do with synonyms as they are not considered in the model. Finally as oxtioned in (Natt och Dag 2006a), there is no guarantee that two requirements at anilar according to the $\sigma($.$) measure are indeed$ related. The method eval doe consider hypernyms and hyponyms (Jackson and Moulinier 2002).

The ReqSimile tool eva ated in this paper is not the only research tool that provides support for requirements tracd bility. Hayes et al. (2007) proposed a REquirements TRacing On-target (RETRO) tool that uses the LSI technique to find similarities between analyzed elements and help the analyst with making traceability links. Lin et al. (2006) proposed a Web-based tool called POIROT that supports traceability across distributed heterogeneous software artifacts. A probabilistic network model is used by POIROT to generate traces between requirements, design elements, code and other artifacts stored in distributed third party case tools.

The second method evaluated in this study uses searching and filtering functionalities provided by Telelogic DOORS tool (IBM 2010b). According to the product documentation, 
the " "finding text in a module" function can search for all the objects that contain a specific search string. The tool displays the options of the search that have already been set in the search window. The possible options are: (1) highlight matches, (2) match case and (3) use regular expressions. To change the search options, the Advanced tab in the same window has to be used. Additionally, it is possible to select the attributes included in the search, for example object heading or object text. The tool provides UNIX-style regular expressions support when searching for text. For example, using c.t will search for all three letter words that start with c and end with t. Using 200[123] will search for either 2001, 2002, or 2003. Subjects during the experiment can also use filters to control what data is displayed on the screen. The tool provides two types of filters: simple and advanced. Simple filters can be used to filter: (1) the contents of every attribute of type text or string, (2) object heading number, (3) the content of any column or the value of a single attribute of any type. Additionally, it is possible to filter on the basis of whether the object has links or is either the current object or a leaf object. Using advanced filters gives the possibility to combine simple filters to create complex filters, specify filter options that control what is displayed.

Using filters requires more steps than using the searching functionality. First, the filter has to be defined and its attributes have to be set. After this step, the user has to define if the filter should match case option or regular expressions. Finally, it is possible to filte only objects that have certain types of links, such as in-links, objects that are leafs and the content of columns. While using advanced filters, it is possible to combine defined simple filters by using And, Or and Not to combine them into logical e ressio It is also possible to control the output of applying a filter. The possible ptr show ancestors or (2) show descendants of the object that match the filte criter ano(3) display all table cells and their contents regardless of whether they m crit tho criteria. According to the product documentation, the Telelogic DOORS tool oes int provide any auto-completion, stemming or proximity search options. Exclusion searon.. are possible by combining two simple filters where one is negated by using th CIOT" logical expression. ${ }^{2}$

The requirements consolidation task can, in a broad perspecti e, be considered as the more general task of negotiating the scope of the futu project with multiple stakeholders. Assuming this broad definition of the task, we dis supporting this negotiation process. Fricker et al. (Fricker al 20 ) propose a negotiation process, called handshaking with implementatio pr process has been used to communicate requirements effectively, evenin ratio, where almost no written requirements exist and where distance separates the ston from the developers. The architectural options are used in this case to unders d d rements and make implementation decisions that will create value. Sommervi and yer (1997) proposed a viewpoint-based approach to requirements engineer which be used to structure the requirements description and expose conflicts betw in mequirements. As systems usage is heterogeneous, viewpoints can organize di rent types of information needed to specify the system and by that help to structure the p ycess of requirements elicitation. Breaux (Breaux 2009) uses grounded theory to analyze regulations and legal documents for the purpose of ensuring that the software system to be built is demonstrably compliant with relevant laws and policies.

2 The information about searching and filtering functionalities has been based on the manual for DOORS version 8.3. The instruction of how to use Telelogic Doors for linking used in this experiment is available at http://fileadmin.cs.lth.se/serg/ExperimentPackages/ReplicationReqSimile/ HelpSheetDoors .pdf 
4 Experimental Design

The two main objectives of this study, presented in Section 1, can be further refined to the following research questions:

Q1: Can significant differences between the assisted and the manual methods that were achieved in the original experiment be confirmed in a replicated experiment where the original manual method is replaced with a keyword searching and filtering tool?

Q1a: Is the assisted method more efficient in consolidating two requirements sets than the manual method (where efficiency is calculated as the number of analyzed requirements)?

Q1b: Is the assisted method more correct in consolidating two requirements sets by assigning more correct links than the manual method (correctness is calculated as a number of correctly linked requirements)?

Q1c: Does the assisted method help to miss fewer requirements links than the manual method?

Q2: Is there any difference between the original and replicated experiment sessions for th same method?

Q2a: Is there any difference in the results for the assisted method betweep he o ginal and the replicated experiments?

Q2b: Is there any difference in the results for the manual methods bety en th on inal and the replicated experiments?

The aim of Q1 is to assess if the results obtained in the original ex arip ent holds even if one of the tools is changed. The research question Q1 is divird into three sub-questions, where each of them is explicitly addressing various qual a the consolidation process. Question Q2 aims to assess the difference between twe periments. The possible differences provide valuable input regarding the natur of the consolidation task and the subjects used in both experiments.

The central part of the requirements consoli ation ask is finding similarities between the two sets of requirements as describ in ection 3. The methods evaluated in the experiment were implemented in to ts (1) ReqSimile (Natt och Dag et al 2006) and (2) Telelogic Doors (IBM 2010a). As s ntio in Section 1, the goal of the study is not to evaluate the tools in general, but to methods that they provide. The planning phase was based on the original perin nt (Natt och Dag et al 2006) and, when possible, the original material is re and ex designing experiments presented by Wohl et and o00). In order to draw more general conclusions, the authors put additional effort to minimizing the difference between this experiment design and the original experiment d sign.

Since most of the design has been reused from the original experiment, the evaluation of the experiment design for the replication sake was limited to checking additional changes. The changes concern questionnaire improvements and new instructions regarding the use of the Telelogic Doors tool (IBM 2010a). The experiment design was evaluated by an additional researcher, experienced in conducting empirical research in software engineering, before executing the experiment. The same researcher participated in the pilot study where both tools were used to find similar requirements and create links between them. Comments and suggestions regarding readability and understandability of the laboratory instructions 
Fig. 1 The process of using the support tool for requirements consolidation.

were given and later implemented. Finally, since the rearire nents used in this experiment were the same as in the original experiment, the correct ans er to the consolidation task remained unchanged ${ }^{3}$.

Similarly to the original experiment, this repludy was also conducted in the form of a laboratory experiment, since it captures the co solidation problem in an untainted way. Fig. 1 depicts the conceptual solutian $f^{\prime}$ dation activity. To the left in Fig. 1, two requirement sets A and B are shown. hey present two consecutive submissions of requirements specifications from the sa ke customer. We can also assume that the earlier specification is set $\mathrm{A}$ in this case, and at have already been analyzed and the result from the analysis is available in cen requirements database. The subjects use one of the tools, either Telelogio ars for hanual method (IBM 2010a) or ReqSimile for the assisted method (Natt och ag a 06 ), to find requirements in the set B that were already analyzed in the set A and to ark them by assigning a link between them. The output of the process is shown to the right ff Fig. 1. The subset A' comprises all requirements that are not linked to any requirement in the set $\mathrm{A}$. The subset $\mathrm{B}^{\prime}$ represents all new requirements that have not previously been analyzed. Finally, there is the subset $\mathrm{C}$, which comprises all requirements in the new specification that previously have been analyzed. The analyst would then send the requirements in set B' to the experts for analysis. The experts are thus relieved from the burden of re-analyzing the requirements in subsets A' and C.

\footnotetext{
3 The experiment pack can be accessed at http://fileadmin.cs.lth.se/serg/ ExperimentPackages/ReplicationReqSimile/Experiment.rar
} 
4.1 Goals, Hypothesis, Parameters and Variables

The variables in this experiment were kept unchanged from the original study (Natt och Dag et al 2006). They can be grouped into independent, controlled and dependent:

- The independent variable is the method used in the experiment. The two methods compared are manual and assisted.

- The controlled variable is the experience of the participants. In order to analyze the individual experience of the subjects, a questionnaire was used.

The dependent variables are:

$\mathrm{T}$ - time used for the consolidation

$\mathrm{N}$ - the number of analyzed requirements

$N_{c l}$ - number of correct links

$N_{i l}$ - number of incorrect links

$N_{c u}$ - number of correctly not linked

$N_{i u}$ - number of missed links (incorrectly not linked)

These dependent variables are used to analyze the hypotheses. The number of analyze requirements is used in case the subjects are not able to analyze all requirements, which will affect $N_{i u}$ and $N_{c u}$. The hypotheses for comparing the manual and the assisted methou remain unchanged from the original experiment design. The rationale of the pra osed potheses is based on the following theory regarding using the assisted method Tha rsi led method provides a list of candidate requirements ranked by their similarity degre to currently analyzed requirement. As a result the requirements analysts hovir $N$ only a subset of all possible combinations, for example the top ten candidate inks. Thus we state a hypothesis that the assisted method can help to analyze requirements far oreover, since the most similar requirements are placed next to each other on list of candidates it is easier to read all potential candidates that exhibit high degr of similarity. The result is expected to be an increased number of correct links, betrecisin and accuracy. Finally, the sorting according to lexical similarity should, in $\delta$ opinion, help to miss fewer correct requirement links, since there is a high probab ans lith possible links to analyze will be show in the top 10 or 20 candidate requiremen Pres hted below are six null hypotheses:

$\left(H_{0}^{1}\right)$ The assisted method results in the sat num of requirements analyzed per minute, $\mathrm{N} / \mathrm{T}$, as the manual method.

$\left(H_{0}^{2}\right)$ The assisted method results the s pe share of correctly linked requirements, $N_{c l} /\left(N_{c l}\right.$ $+N_{i u}$ ), as the manual metl

$\left(H_{0}^{3}\right)$ The assisted method re alts in the same share of missed requirements links, $N_{i u} /\left(N_{c l}+\right.$ $N_{i u}$ ), as the manual method.

$\left(H_{0}^{4}\right)$ The assisted method results in the same share of incorrectly linked requirements, $N_{i l} / \mathrm{N}$, as the manual method.

$\left(H_{0}^{5}\right)$ The assisted method is as precise, $N_{c l} /\left(N_{c l}+N_{i l}\right)$, as the manual method.

$\left(H_{0}^{6}\right)$ The assisted method is as accurate, $\left(N_{c l}+N_{c u}\right) /\left(N_{c l}+N_{i l}+N_{c u}+N_{i u}\right)$, as the manual method. 
Table 1 The number of years of industrial experience in software development for pairs of the subjects that participated in this replication. The remaining pairs of subjects exhibited no industrial experience for both pair members. The letter A indicates that a pair of subjects used the assisted method while the letter $\mathrm{M}$ indicates that a pair of subjects used the manual method. The IDs (Mx and Ay) are consistent with Table 5. The rows highlighted gray indicate data points that were removed from the analysis (outliers).

\begin{tabular}{lll}
\hline Pair of subjects & $\begin{array}{l}\text { Experience of the first subject in the } \\
\text { pair (in years) }\end{array}$ & $\begin{array}{l}\text { Experience of the second subject in } \\
\text { the pair (in years). }\end{array}$ \\
\hline A1 & 0.5 & 1.5 \\
A3 & 1 & 1 \\
A7 & 1 & 2 \\
A10 & 0 & 1 \\
A11 & 0.5 & 1 \\
M4 & 1 & 2 \\
M5 & 0.5 & 0 \\
M6 & 0.25 & 0 \\
M7 & 0.5 & 1 \\
M8 & 0.25 & 1.5 \\
\hline
\end{tabular}

Since the subjects may not use exactly the same time for the task, the performan e is ormalized as the number of analyzed requirements divided by the total time spe on he consolidation task (in minutes).

\subsection{Subjects}

In this study, a different set of subjects compared to the rig riment, although from the same kind of population was used. The sample ingles perteipants of the course in Requirements Engineering at (Lund University 2011a The course is an optional masterlevel course offered for students at several enginee atg ro and electrical engineering. It gives 7.5 ETCS p ints (CTS 2010) which corresponds to five weeks full time study. Although the mas a mandary part of the course, the results achieved by the subjects be influd on their final grade from the course. The students were between 24 and $4 k$ ars $y$ with an average of 27 years. There were 4 female and 41 male students. Befo co acting the experiment, the subjects had been taught requirements engineering rmin gay and had gained practical experiences through their course project. The from the difference in English reading and wi in mo small, varying from "very good knowledge" for the majority of subjects to "fluen knowledge" for some of them. When it comes to the industrial experience in software devel sment of the subjects, most of them reported no experience at all (28 out of 45 students). Among the subjects that reported any degree of industrial experience, the length of the experience varied between three months and two years with an average value of 11 months. The analysis of industrial experience in pairs of subjects revealed that ten pairs had varying degrees of industrial experience which was not always equal. The analysis of the experience of both pair members is presented in Table 1 . The difference in experience varied between three months and 15 months with an average value of nine months. The impact of the industrial experience on the results achieved by these subjects is outlined and discussed in detail in Section 7.1 and Table 7. 
Table 2 The experience and the roles of the subjects that participated in the replication from the course that the requirements originate from. The letter A indicates that a pair of subjects used the assisted method while the letter $\mathrm{M}$ indicates that a pair of subjects used the manual method (the numbers are consistent with Table 5). The rows highlighted gray indicate data points that were removed from the analysis (outliers).

\begin{tabular}{lll}
\hline Pair of subjects & Role of the first subject in the pair & Role of the second subject in the pair \\
\hline M2 & Have not taken the course & Tester \\
M3 & Have not taken the course & System group member \\
M4 & Development Manager and Developer & Test Manager and Tester \\
M5 & Tester & Have not taken the course \\
M6 & System Group Member & Project Manager \\
M7 & Have not taken the course & Developer \\
M9 & System Group Member & Developer \\
M10 & Development Manager & Project Manager \\
M11 & Have not taken the course & System Group Member \\
\hline A1 & Project Manager & Have not taken the course \\
A2 & Project Manager & Tester \\
A3 & Test Manager and Tester & Developer \\
A4 & Developer & Tester \\
A5 & Have not taken the course & Have not taken the course \\
A6 & Project Manager & Have not taken the course \\
A7 & Developer & \\
A12 & Developer & \\
\hline &
\end{tabular}
subjects have not taken the course that the requirements origi while the rest had taken the course and acted in various roles during the prect of $f$ the course.

Next, the roles taken in the course that the requir ments orirs formed by subjects were analyzed. For 9 pairs, outlined Table 2, the pairs are formed by an inexperienced person and an experienced person for he ourse. This may have a positive impact on the task, since the more experienced ferson tan help the inexperienced person

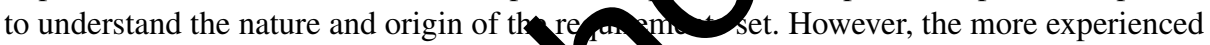
person can bias the consolidation tas by ingin. knowledge about the requirements sets and possible similar requirements frok he $c$ vrse. The remaining seven pairs represented experience from various roles incluo $\mathrm{ng}$ : manager, project manager and ter. $\mathrm{O}$ one pair had the same experience from being a developer, in other cases ales tan the course project did not overlap.

When it comes to the expe in analyzing and reviewing requirements, $80 \%$ of the subjects declared to ha experience only from courses. Among the remaining 20\% of the subjects, one pair (M $\mathbf{}$ had experience from both courses and industry (less than one year). In this case, the second pair member had only experience from courses. Furthermore, in cases (A1,A3 and A11), one pair member reported both experience from courses and less than a year of industrial experience in analyzing and reviewing requirements. In all three cases, these participants were paired with subject reporting only experience from the courses. Finally, in the case of pair M8, one member reported more than one year of industrial experience, while the other pair member reported no experience at all. The analysis of the results achieved by these subjects in relation to their experience is discussed in Section 7.1. 
Table 3 The treatments and tools used in the original and the replicated experiments.

\begin{tabular}{|l|l|l|l|l|}
\hline & \multicolumn{2}{|c|}{ Original experiment } & \multicolumn{2}{c|}{ Replicated experiment } \\
\hline Treatment & Assisted method & Manual method & Assisted method & Manual method \\
\hline Tool & ReqSimile & ReqSimileM & ReqSimile & Telelogic Doors \\
\hline
\end{tabular}

A further question concerned the subject's experience with the tool that implements the manual method, that is Telelogic Doors. The analysis indicated that $91 \%$ of subjects reported no experience with Telelogic Doors and that they had never heard about the tool. Although four persons have heard about the tool, they have never used it. We can conclude that the subjects are homogenous in this matter and that we can exclude this threat from aspects influencing the results.

\subsection{Treatments}

The treatments of the experiment are the methods used in supporting the requirements cop solidation task. The assisted method is implemented in the ReqSimile tool using linguistio engineering to calculate the degree of similarity between requirements by lexical similarm as a way of approximating semantic similarity (Natt och Dag et al 2004) (for ms e do ils see Section 3).

The other treatment is the manual method which comprises searching ar filt ing unctionalities provided by the Telelogic Doors tool (IBM 2010a). The goal of t exp riment is not to compare the two tools in general, but the functionality that they rovide w support the requirements consolidation task. The objects used in the original and re icated experiment are listed in Table 3. Compared to the original experiment one of the tools was kept unchanged while the second one was changed. The chare constituting ReqSimileM from the original design (Natt och Dag et al 2006) br Telelogid Doors (IBM 2010a) for the manual method. More information regarding tools sed in the replication can be found in Section 4.5.

\subsection{Requirements}

Two requirements sets were reused moriginal experiment. The requirements specifications were produced as a pa of a urse "Software Development of Large Systems" (Lund University 2011b). mprises a full development project, including: requirements specification, te ts aration, high-level design, implementation, test, informal and formal reviews and acce tance testing. At the end of the course, the students deliver a first release of the controller oftware for a commercial telecommunication switch board. Two requirements specifications were randomly selected from the course given in years 2002 and 2003. The requirements have been specified in use case style or features style (Lauesen 2002), and all are written using natural language. Two requirements sets containing 30 and 160 requirements respectively, were imported to ReqSimlieA and Telelogic Doors. An example of requirements from the specification comprising 30 requirements is depicted in Table 4. However, the requirements were neither written by a native English language writer, nor given to a native English language speaking, experienced requirements analyst for editing and rephrasing. 
Table 4 Example requirements from the specification comprising 139 requirements.

\begin{tabular}{|c|c|c|c|c|}
\hline Key & Id & Type & Selection & Description \\
\hline 3 & Scenario13 & Functional & $\begin{array}{l}\text { Service: } \\
\text { Regular } \\
\text { call }\end{array}$ & $\begin{array}{l}\text { Regular call-busy Actors: A:Calling subscriber, } \\
\text { B:Called subscriber, S:System Prerequisites: Both } \\
\text { A and B are connected to the system and are not } \\
\text { unhooked. Step 13.1. A unhooks. Step 13.2. S starts } \\
\text { giving dial tone to A Step 13.3. A dials the first digit } \\
\text { in B_s subscriber number Step 13.4. S stops giving } \\
\text { dial tone to A. Step 13.5. A dials the remaining three } \\
\text { digits in B_s subscriber number Step 13.8. S starts } \\
\text { giving busy tone to A Step 13.9. A hangs up Step } \\
\text { 13.10. S stops giving busy tone to A }\end{array}$ \\
\hline 80 & SRS41606 & Functional & $\begin{array}{l}\text { Service: } \\
\text { Call for- } \\
\text { warding }\end{array}$ & $\begin{array}{l}\text { Activation of call forwarding to a subscriber that has } \\
\text { activated call forwarding shall be ignored by the sys- } \\
\text { tem. This is regarded as an erroneous activation, and an } \\
\text { error tone is given to the subscriber. (Motivation: To- } \\
\text { gether with SR41607, avoids call forwarding in closed } \\
\text { loops) }\end{array}$ \\
\hline 111 & SRS41804 & Functional & $\begin{array}{l}\text { Service in- } \\
\text { teraction }\end{array}$ & $\begin{array}{l}\text { The service call forwarding shall be deactivated if a } \\
\text { customer removes either the subscriber from whif } \\
\text { calls are forwarded or the subscriber to which calls ar } \\
\text { forwarded. }\end{array}$ \\
\hline
\end{tabular}

other tool was changed. The tool that implements the assistemethod, that is ReqSimile (Natt och Dag 2006b), was kept unchanged. The user in Fig. 2. The left side of the top pane of the window presents a list requirements. The data has already been pre-processed by the tool so the user on start analyzing requirements for similarities. Selecting a requirement (1) makes the ront's details display on the right (2) and a list of similar requirements in the other (t app or in the bottom pane (7), sorted on the similarity value (3). Requirements that been linked in the set analyzed requirements are highlighted using arotho (gray color (6). Requirements that have been linked to the currently selected require nts are highlighted using another (green) color (5). Unlinked requirements are not h, (8). Links can be made between the selected requirement (2) and the requirem wit the associated link button (4). Once a requirement has been selected (1), the has form two operations, click on the requirement that is supposed to be linked an cly button "link" to make the link.

The second tool, describ d by Natt och Dag et al. (2006) as ReqSimileM was changed in this experiment to Telelog Doors (IBM 2010a). The user interface of Telelogic Doors is shown in Fig. 3. The two sets of requirements were opened in Doors from separated modules and placed next to each other on the screen. Fig. 3 illustrates one of the requirements sets opened in a module. This orientation is similar to ReqSimile's view and enables easy visual comparing between the two sets of requirements. The finding and filtering capabilities were used in Telelogic Doors to perform the consolidation task. These capabilities can be accessed respectively from the Edit menu and the Find command or the Tools menu and the Filters command. The subjects were given detailed instructions with screen-shots of each step and each dialog window that was related to finding and 


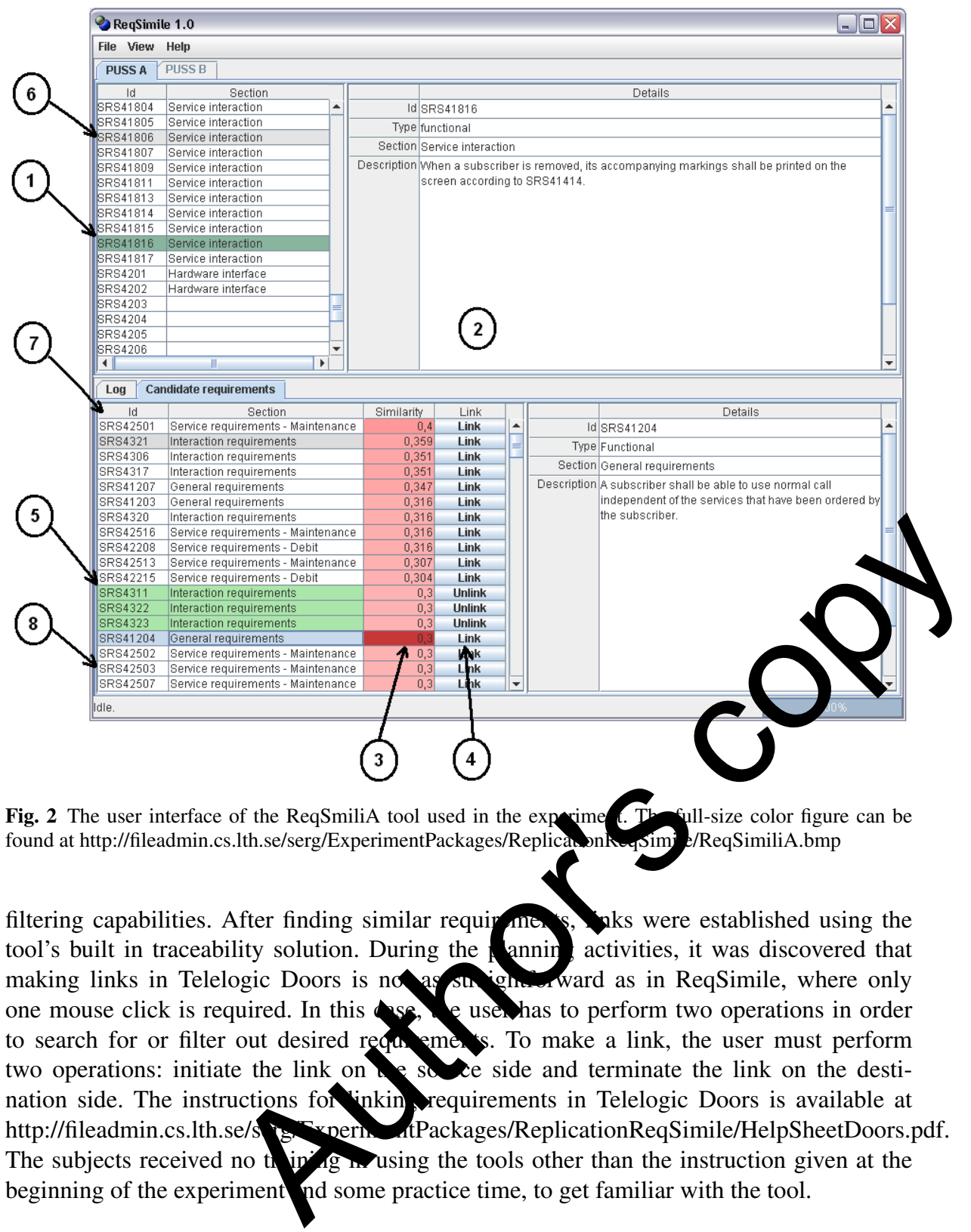

\subsection{Correct Consolidation}

To enable measurement of the subjects' accuracy of linking requirements that are semantically similar, the original key for assigning correct links has been reused. This original key was created by the first author of the original experiment article (Natt och Dag et al 2006), having many years of experience from this course in various roles. It is therefore justifiable to consider this key as one provided by an expert in the domain. The key was created a priori 


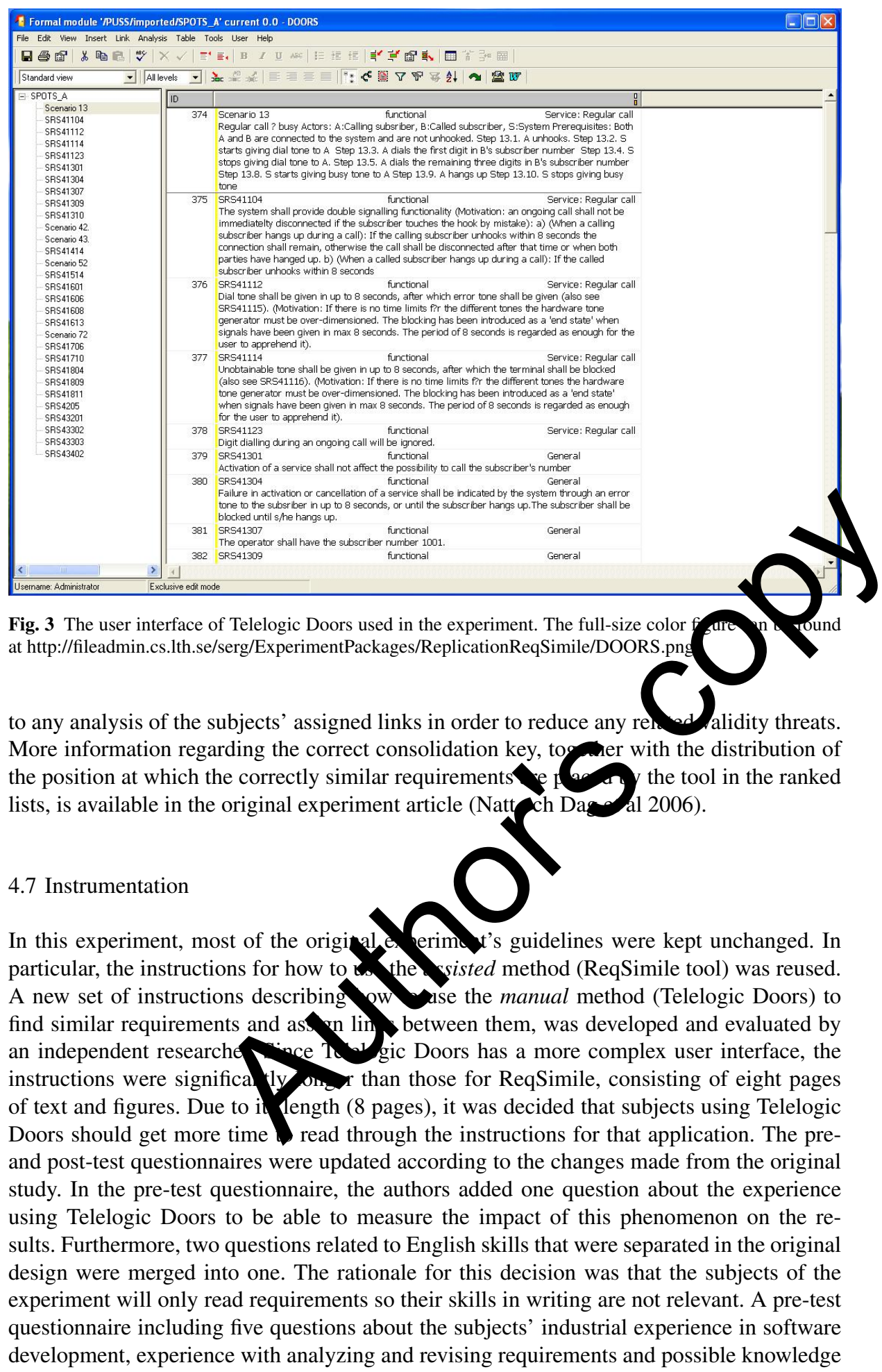


and skills in Telelogic Doors was prepared. Before collecting the data, an additional experienced researcher evaluated the questionnaire to check the understandability of questions and their relevance for this study.

Due to a limited number of available computers in the laboratory room, the subjects were asked to work in pairs for the experiment. This deviates from the original experiment design, where subjects were performing the task individually and demands additional analysis to ensure that groups were formed equally. Some changes were also made to the post-test questionnaire. The original questions regarding (1) the time spent on the consolidation task, (2) the number of finished requirements, (3) the number of found duplicates, similar and new requirements and (4) the usefulness of used methods were kept unchanged. Moreover, two questions about the scalability of used methods and possible improvements were kept unchanged comparing to the original experiment design. ${ }^{4}$.

\subsection{Data Collection Procedure}

The data collection procedure was kept as similar as possible to the original experiment design. The subjects were given the introduction and problem description by the moderato After the introduction, subjects were given some time to read through the assigned tool's instruction and make themselves familiar with its user interface. At this stage, the grour assigned to work with Telelogic Doors were given some extra time (approxima ely 10 minutes) since the tool interface was more complex and the instruction was longd on of the important changes here is that the subjects answered pre-study test righ beto s ting the task. The results of the pre-study survey were analyzed afterward and pre sented in Section 4.2. Next, the subjects were asked to work for 45 minutes on e conpondation task. The results from the actual experiment were collected by analyzing the for ation recorded in the tools about the number of links made and requirements anlyzed by the subjects. The experimental results were also checked against the resul of to ensure consistency. The post-questionnaire was asked after performing thask.

\subsection{Validity Evaluation}

As for every experiment, questions abo the lidity of the results must be addressed. Threats to validity are presented and scus using the classification of threats to conclusion, internal, construct and exter l va dity as proposed by Wohlin et al. (2000).

Conclusion validity. In order not ye too low power of the statistical tests, parametric tests (t-test) were used af having ve tigated the normality of the data.

Subjects were selected fror same education program to limit their heterogeneity. Moreover, the questionnaire out the experience of subjects from industry, experience from the course where requiremes originate from and experience in reviewing requirements was used to assess the heterogeneity of the subjects in these aspects. However, the threat related to the fact that subjects were asked to work in pairs may impact the conclusion validity. It affects in particular the random heterogeneity of subjects, since created pairs may manifest differences in industrial experience or experience from the previous courses. We discuss this threat in the analysis of the pre-study questionnaire results in Section 4.2

\footnotetext{
4 Both questionnaires are available at http://fileadmin.cs.lth.se/serg/ExperimentPackages/ ReplicationReqSimile/PreTest.pdf and http://fileadmin.cs.lth.se/serg/ ExperimentPackages/ReplicationReqSimile/PostTest.pdf
} 
and the results achieved by the subject in relation to their experience in Section 7.1. The way how the subjects took seats in the laboratory room and thus the way how they were assigned to the methods can also be questioned here. As pointed out by Wilkinson et al. (1999), random assignment is sometimes not feasible in terms of the control or measure of the confounding factors and other source of bias. Elements outside the experimental setting that may disturb the results were minimized. Students were not interrupted during the experiment sessions and no significant noise was present. In order to minimize random irrelevance in experimental setting, the experiment moderators ensured that any discussions in pairs of subjects should be made as quietly as possible.

Although all subjects have taken the same education program for 2.5 years, the individual differences in industrial experience, experience in the course from which the requirements originate, and knowledge of English may affect the results. The searching for a specific result threat was addressed by not notifying the subjects which method is supposed to perform better than the other. The threat to the reliability of measurements is addressed by reusing the original measurements for the replication case. Moreover, all subjects received the same instruction for using the treatments which helps to standardize the application of treatments to subjects. Finally, the error rate of the significance level and the use of Bonferroni correction (Arcuri and Briand 2011) are among the threats to conclusion validity. The possibility of using the Bonferroni correction to adjust the level of significance is discussed in Section 7.

Internal validity. In this case, threats related to the history, maturation etc. 1 ave $t \mathrm{be}$ mentioned. The history threat to internal validity is minimized by applying one trea men to one object. Both days when the experiment sessions were held where norm 1 wo in tays not followed by any holidays (the first session took place on Tuesday a se ond session on Friday). The maturation threat was minimized by dedicating o (y 45 pinutes for the consolidation task (we assume that the subjects won't get bored by the 45 minutes). The instrumentation threat is addressed in two ways: (1) by reving the original experimentation instrumentation, if no changes were needed, and 2) ng the instrumentation documentation by an independent researcher. However, fnce subio ts were not divided into groups according to the results of the pre-study ques nnaire (the questionnaire has been filled in right before the experiment's execution st stical regression threat can not be as easily addressed as in the original experin ent. I e analysis related to this threat is presented in Sections 4.2 and 7.

The incentives of participants ar no to the experience, an important factor that may influence the results of this stud 4 cco ding to the classification presented by Höst et al. (2005), both the original expernen replication can be classified as I2 E1 where I2 means that the project is artifi 1 (in rms of incentive). The subjects typically have no prior knowledge of the a the requirements sets used in the experiment were de elo en the researcher or borrowed from an industrial organization. The E1 level on th experience scale means that the subjects are undergraduate students with less than three lonths recent industrial experience, where recent means less than two years ago. Although the identical comparison of two I2E1 cases is not present in Höst et al. (2005), the example of two experiments classified as E1 I1 (where I1 means an isolated artifact) shows no significant difference in their outcomes. Moreover, three other pairs of experiments, classified in the same category, also shows the same outcomes (Höst et al 2005).

The selection threat, as in the original design, may influence the results since the subjects are not volunteers and the laboratory session where the experiment was performed is a mandatory part of the course. The social threat to internal validity is addressed since 
the subject had nothing to gain from the actual outcome of the experiment; the grading in the course is not based on results of, or preparation for, the experiment. Unlike the original experiment design, the experiment groups were not separated, however no information about which method is expected to perform better was revealed to the subjects. The possibility of looking at other subjects' results during the experiment execution was minimized by placing the subject in a way that separated each of the two treatments by the other treatment. Compensatory rivalry may be a problem in this case, since the group that will use the open-source solution (ReqSimile) or the commercial solution (Telelogic DOORS) may try to perform better to make their favor type of software win. This threat was addressed by explicitly stating in the beginning of the experiment that there is no favorite or assumingly better method. The potentially more problematic threat is that the subjects had to analyze and link requirements written in English when they had themselves used only Swedish to specify their own requirements in the domain. Further, the participants ability to objectively evaluate their skill in English language is a subject to question. In further work it would be interesting to execute the experiment on a set of native English subjects, also handling the original set of requirement to a native speaker experienced requirements analyst who will edit and rephrase them.

Construct validity. In this case, the theory is that the assisted method implemented in the ReqSimile tool provides better assistance for a particular task than the method implemented in Telelogic Doors. We base this theory on the fact that the assisted method a list of candidate requirements augmented with the degree of lexical similarity. A a re 1 , the analyst can only look at a subset of possible candidate requirements (the mo sim ar requirements, up to a certain threshold) thus saving time required to do the ask. Yor ver, we believe that the list of possible candidates helps the analyst to miss red rements links and increase the precision of making the links. In contrast to th original experiment design, none of the authors have developed any of the tools. However, he riginally mentioned threat related to the awareness of subjects about their oy orrors is still present in this experiment. This threat may have influenced the number o c a d faulty links. Also, as pointed out by Natt och Dag et al. (2006), when subjectronow the time is measured, it is possible that they become more aware of the time sperformance results may be affected.

The level of experience of the subjects, espe ially reviewing requirements, may influence the outcome of the experiment. Thi anstruct validity is addressed by the analysis and discussion of the results chio by bects having experience in reviewing requirements in Section 7. Becaus se 30 and 160 requirements were used by all subjects in both treatments and expenensessions, this may result in a situation where the cause construct is under-rep ented This threat is discussed in Section 7 where alternative designs for this ex ment outlined. Moreover, keeping the requirements sets unchanged opens up the p ssi mor of discussing other factors as well as differences between the original and repl ated experiment to assess their influence on the results. The interaction of different treatm nts threat to construct validity is minimized by involving the subjects in only one study. The differences in the user interfaces and their usability may have influenced the results achieved by the subject. In particular, this threat could influence the numbers of requirements analyzed and links assigned by the subjects. This threat has been addressed in two ways: (1) by providing detailed instructions on how to make links in the tools and by giving the subjects as much time as they requested to get familiar and comfortable with the user interface, (2) by making the user interfaces look as similar as possible by placing the two requirements sets next to each other in Telelogic DOORS. Finally the evaluation apprehension threat is minimized by: (1) clearly stating that the performance of 
the subject has no effect on their grade in the course and (2) by using requirements that were not written by the subjects.

External validity. The largest threat in this category is the number of analyzed requirements. Since only a relatively small number of requirements was analyzed during the experiment, it is hard to generalize the results to large sets of requirements, which often is the case in industry projects (Berenbach et al 2009, Leuser 2009). Using students as subjects is another large threat. Even though the subjects were on their last year of studies, they can be considered as rather similar to an ordinary employee. However, as mentioned by Kitchenham et al. (2002) students are the next generation of software professionals and they are relatively close to the population of interest. Since they participated in the requirements engineering course, they are familiar with the application domain. Finally, the diversity of experience of subject from industry and from analyzing and reviewing requirements, although hindering the conclusion validity, has a positive influence on the heterogeneity of the population sample used in this experiment.

The time spent on the task is also among potential threats to external validity. To analyze 30 requirements in 45 minutes subject should spend on average 90 seconds on each requirement. It remains an open question whether or not this is enough time for the subjects to perform both lexical and semantic analysis. However, this threat was partly addressed b stating at the beginning of the experiment that the subjects don't have to analyze all 30 requirements in 45 minutes (the subjects had to record how many requirements were analyzon and how they browsed the list of candidate requirements).

\section{Experiment execution}

The replication was run in two two-hour laboratory sessions in Janua 20 8 . The first 15 minutes of each session were dedicated to the presentation of problem. During this presentation, the importance of the industrial applicability th the goal of the experiment were stressed. All students were given the sameresentatin. The general overview and differences between the included methods and to 5 were presented without favoring one method over the other. To avoid biasing, no h pores were revealed and it was made very clear that it is not known which approach ill pe form better. After the presentation of the problem, students were given time with the instructions of how to use the tools and report that they are reas, to sta working on the task. The starting time was recorded in the questionnaire as st. nts guired varying times to get familiar with the instructions. After the 45 minutes tin as for the task, subjects were asked to stop, record the number of analyzed ruiren the time, and to fill in the post-questionnaire. The remaining time was for anging experiences and discussion the abouts used. This approach is sim ar original experiment execution described by Natt och Dag (2006). The difference rom the original experiment is that subjects used both methods in both experimental sess nns. Half of the subject pairs in each session (there were two sessions in total) were assigned to the assisted method while the other half to the manual method. The subjects were to use only one method during the experiment and participate in only one of the two sessions. The difference from the original experiment here is that the methods were not separated in different sessions. That is, in each session the pairs using the assisted and the manual methods were mixed.

After the presentation, the subjects were assigned to the methods. Because only one laboratory room could be used for each session and this room did not have enough computers for all subjects (which was not known while planning the experiment), the subjects 
were asked to work in pairs. Each pair was randomly assigned to the method later used for the consolidation task. There were no name tags or other indicators of the method on the laboratory desks when subjects took their seats in the laboratory room. Therefore, subjects could not take a preferable method seat or be attracted by the name on the desk. Subjects were asked to discuss the solutions only within their own pair. Since the nearest group was not using the same method, the possibility of comparing or discussing results was avoided. The subjects were allowed to ask questions of the moderator, if they experiences any problems. Only answers related to the difficulties of using tools were given in a straightforward manner. No answers related to assessing similarity between requirements were given. The material used in the experiments comprised:

- The ReqSimile application with automated support of similarity calculations and a database containing: (1) 30 randomly selected requirements from the first set, (2) all 160 requirements from the second set. These requirements should be browsed through by the subjects.

- The Telelogic Doors application with the same two sets of requirements imported into two separated modules. The application's graphical user interface was set as presented in Fig. 3 in order to make it as similar to the ReqSimile user interface as possible.

- The documentation comprising: (1) An industrial scenario describing the actual chal lenge (one page). (2) A general task description (one page). (3) Detailed tasks space for noting down start and end times (one page). (4) A short FAQ wit questions and answers about the requirements (one page). (5) A screen shot the ol user interface with the description of the different interface elements ile case (one page) or a 8 pages instruction with screen shots from the teps eed to analyze requirements and make links using Telelogic Doors.

- The instruction to the students was as follows: (1) Review as man of th requirements as possible from the list of 30 requirements shown in the tool. Forvach reviewed requirement, decide if there are any requirements in the or set that can be considered identical or very similar (or only a little different) wh ropect o intention. (2) Assign links between requirements that you believe are ide acal or very similar. (3) Note down the start and finish time. (4) When finished, notify th moderator.

Given the experience from the original study (it wa decided to dedicate 45 minutes to the consolidation task. The subjects wer no the lime left for the task 15 and five minutes before the end of the lab ssio Afte spproximately 45 minutes, subjects were asked to stop working on the task unlen they, or any reason, spent less than 40 minutes on the task. All students were asked to $\mathrm{h}$ in post-test questionnaire described in Section 4.7. Apart from noting the finish time nd the number of analyzed requirements, subjects were also asked to asses of used methods in terms of the given task and, if applicable, propose imp ves Right after executing the experiment, it was known which data points had to be i noved due to tool problems or subjects' attitude. Three groups had problems with the tools which resulted in loss of data and one group performed unacceptably analyzing only three requirements during 45 minutes and making only two links. These four groups were treated as outliers and removed from the analysis.

\section{Experiment results analysis}

In this section, methods of analyzing the results are described. In order to keep the procedures as similar to the original experiment design as similar as possible, the same statistical 
Table 5 The results from measuring dependent variables. The rows highlighted gray indicate data points that were removed from the analysis (outliers).

\begin{tabular}{|c|c|c|c|c|c|c|c|}
\hline $\begin{array}{l}\text { Pair of sub- } \\
\text { jects }\end{array}$ & $\begin{array}{l}\mathrm{T} \\
(\mathrm{min})\end{array}$ & $\mathrm{N}$ & $\begin{array}{l}\text { Links as- } \\
\text { signed }\end{array}$ & $\begin{array}{l}\text { Correctly } \\
\text { linked } \\
\left(N_{c l}\right)\end{array}$ & $\begin{array}{l}\text { Correctly } \\
\text { not linked } \\
\left(N_{c u}\right)\end{array}$ & $\begin{array}{l}\text { Incorrectly } \\
\text { linked }\left(N_{i l}\right)\end{array}$ & $\begin{array}{l}\text { Missed } \\
\left(N_{i u}\right)\end{array}$ \\
\hline M1 & 38 & 12 & 6 & 1 & 4 & 5 & 6 \\
\hline M2 & 41 & 13 & 10 & 5 & 4 & 5 & 3 \\
\hline M3 & 46 & 30 & 15 & 11 & 10 & 4 & 9 \\
\hline M4 & 44 & 13 & 10 & 5 & 4 & 5 & 3 \\
\hline M5 & 45 & 18 & 16 & 8 & 11 & 8 & 12 \\
\hline M6 & 48 & 20 & 5 & 2 & 6 & 3 & 9 \\
\hline M7 & 45 & 22 & 21 & 6 & 6 & 15 & 8 \\
\hline M8 & 44 & 19 & 9 & 4 & 7 & 5 & 8 \\
\hline M9 & 46 & 19 & 15 & 7 & 5 & 8 & 5 \\
\hline M10 & 45 & 16 & 9 & 4 & 4 & 5 & 5 \\
\hline M11 & 45 & 18 & $?$ & $?$ & $?$ & $?$ & $?$ \\
\hline A1 & 41 & 14 & 13 & 5 & 5 & 8 & 5 \\
\hline $\mathrm{A} 2$ & 45 & 20 & 25 & 9 & 4 & 16 & 3 \\
\hline A3 & 49 & 18 & 19 & 5 & 3 & 14 & 6 \\
\hline A4 & 45 & 13 & 25 & 5 & 0 & 20 & 3 \\
\hline A5 & 50 & 20 & 15 & 8 & 4 & 7 & 4 \\
\hline A6 & 50 & 21 & 19 & 6 & 3 & 13 & 6 \\
\hline A7 & 29 & 3 & 11 & 1 & 0 & 12 & 0 \\
\hline A8 & 44 & 30 & $?$ & $?$ & $?$ & $?$ & \\
\hline A9 & 34 & 30 & 23 & 13 & 7 & 10 & \\
\hline A 10 & 41 & 30 & 16 & 12 & 8 & 4 & \\
\hline A 11 & 50 & 23 & 19 & 7 & 7 & 12 & \\
\hline A12 & 35 & 30 & 20 & 13 & 8 & 7 & \\
\hline
\end{tabular}

methods were used to test if any of the null hypothese can Additional analysis was also performed in order to assess if working in pirs infue ces the performance of subjects. Hypotheses were analyzed separately, while of relations and accumulated results are presented in Section 7. The standard t-test hron as the data was confirmed to have a normal distribution. Just as in the orig tal ex eriment from which requirements were reused, in this experiment one anslyz ent can be linked to several others. The results from measuring dependen va bles be found in Table 5. Subjects that used ReqSimile are marked with the letter A s an bbreviation of the assisted method), and subjects that used Telelogic Doors with (as an abbreviation of the manual method). The dependent variables are descibed is Section 4.1. Table 5 presents the results from both experimental sessions ( $\mathrm{Tu}$ hey and ari ay sessions).

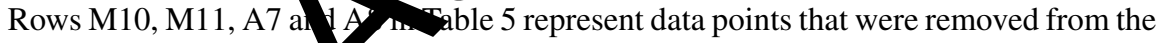
analysis. The pair M10 was moved from the results due to the inconsistency between the results stated in the post-task uestionnaire and the results saved in the tool. The pair M11 was removed due to loss of data. Similar problems caused the authors to remove group A8 from the analysis since the links were not saved in the tool. Finally, group A7 was removed due to their lack of their commitment to the task.

The time spent on the task is presented in column 2 of Table 5 . The results for the number of finished requirements, derived from the post questionnaire and confirmed with the results recorded in the tool used, are listed in column 3. Next, other dependent variables values are presented in the remaining columns. The values were calculated based on the results saved in the tools and from the answers to the questionnaires questions. 


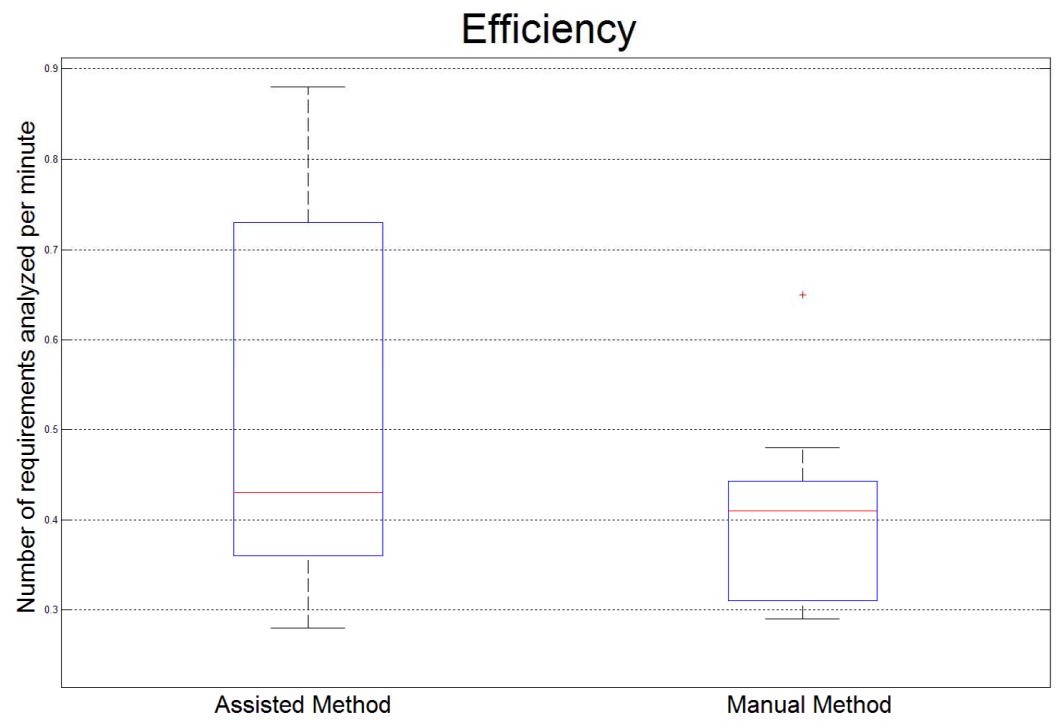

Fig. 4 The results for the number of analyzed requirements.

The results for the number of analyzed requirements per minute are do olct as box plot in Fig. 4. It can be seen that there is no statistically significant differend in th numroer of analyzed requirements between the manual and the assisted metho The group that used the manual method analyzed on average 0.41 requirements per minut whil the group that used the assisted method analyzed on average 0.51 requirementsper minute. In this case, we observe that the medians are most likely equal, while the lo er upper quartiles values differ significantly. The t-test gave a p-value of 0.20 whin gives $\mathrm{y}$ basis to reject the null hypothesis $H_{0}^{1}$. The notches of the box plot overlap. 5 summarize, the assisted method turned out not to be more efficient in consolidatinom tho quirements sets than the manul method (research question Q1a).

The results for the number of correst lip subjects are depicted in Fig. 5. The group that used the assisted method orrec assigned on average 58\% of the links that the expert assigned, while the gre tho used the manual method correctly assigned on average $43 \%$ of the correct link Tho medians differ significantly from $61 \%$ for the assisted method to around $42 \%$ or the anual method. The t-test gave in this case the pvalue 0.013 which makes assib to eject hypothesis $H_{0}^{2}$. Thus, we can state a positive answer to research questit as asisted method is superior to the manul method when consolidating two req rements sets when measured by which method delivers more correct links.

To address hypothesis $H_{0}^{3}$, requirements analyzed by each pair of subjects were reviewed, and the number of links that should have been assigned but were not, was calculated. In the case when subjects did not analyze all requirements, only requirements that had been analyzed were taken into consideration. Each pair of subjects stated in their post-test questionnaire how many requirements were analyzed and how they had worked through the list of requirements. This information was used to correctly count the number of missed links. The results are depicted in Fig. 5. The group that used the assisted method missed on average $41 \%$ of the links, while the group that used the manual method missed on average 


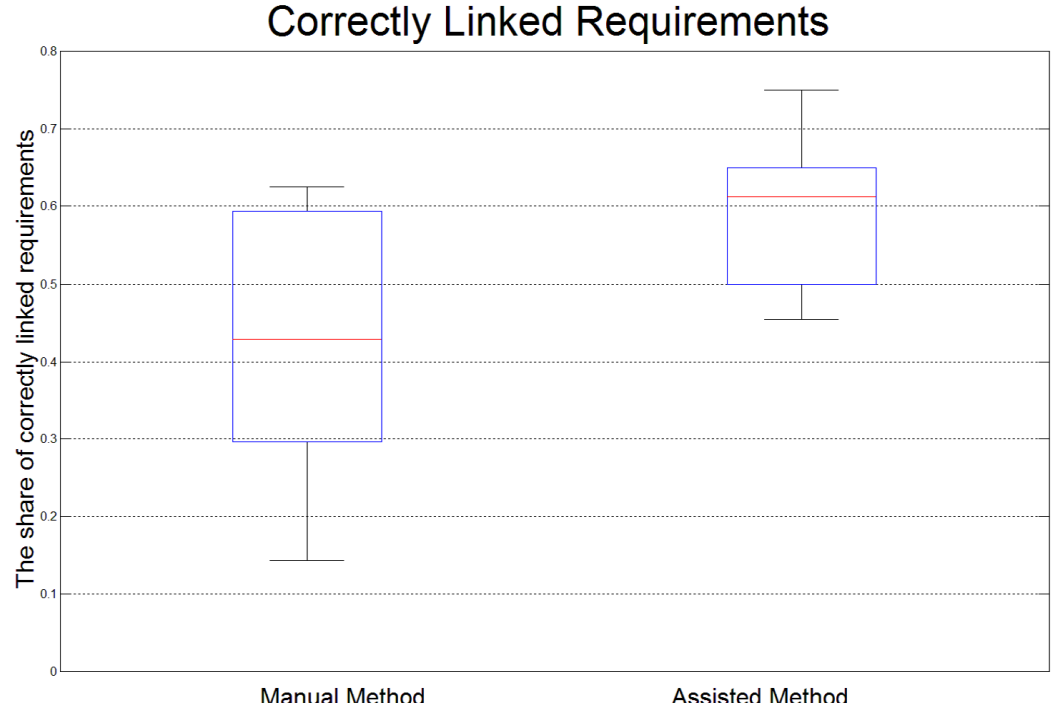

Fig. 5 The results for the share of correctly assigned links.

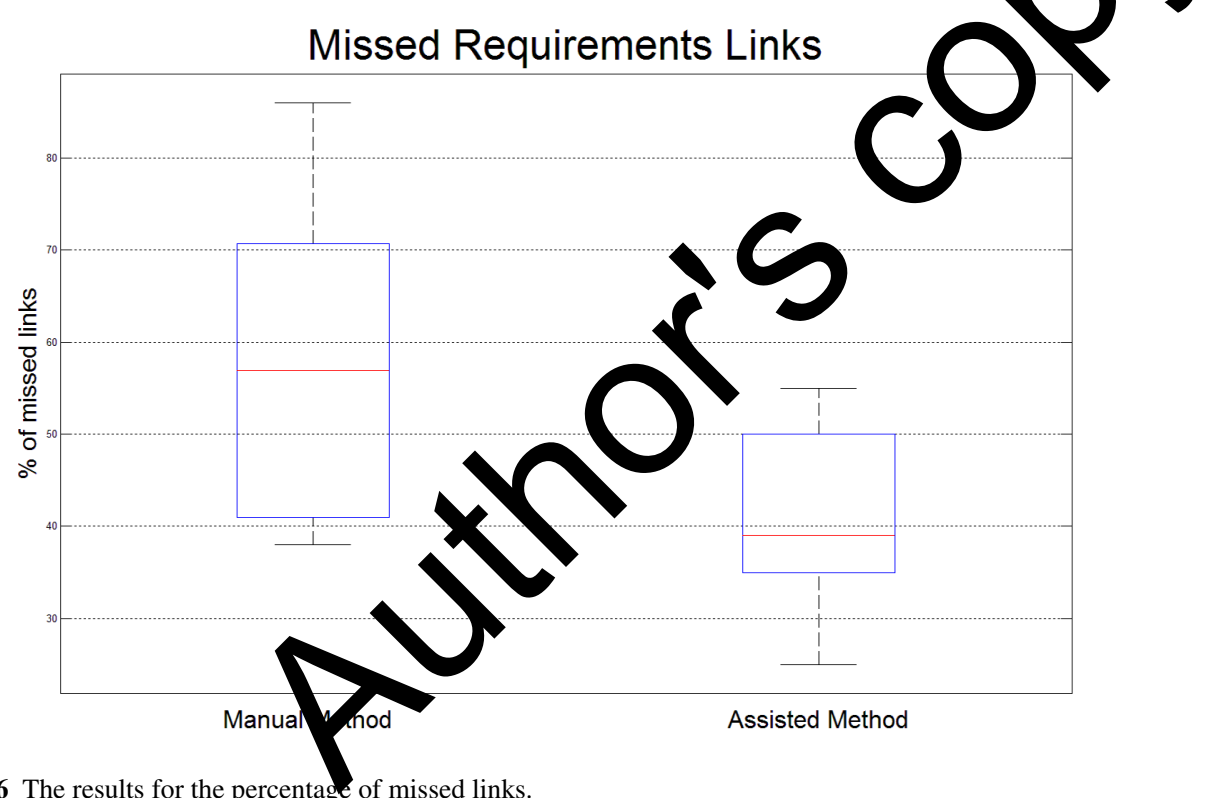

Fig. 6 The results for the percentage of missed links.

$57 \%$ of the links. The medians in this case are $38 \%$ for the assisted method and $57 \%$ for the manual method. The t-test gives a p-value of 0.0207 which means that we can reject $H_{0}^{3}$ and confirm the original experiment's conclusions by making the conjecture that the assisted method helps the subjects to miss significantly fewer requirements links than the manual method (research question Q1c). 
Table 6 The results of the t-tests for original and replicated experiments.

\begin{tabular}{lll}
\hline Hypotheses & $\begin{array}{l}\text { The p-values in the original } \\
\text { study (Natt och Dag et al 2006) }\end{array}$ & The p-values in this replication \\
\hline$H_{0}^{1}$ Efficiency & 0.0034 & 0.20 \\
$H_{0}^{2}$ Correct links & 0.0047 & 0.013 \\
$H_{0}^{3}$ Missed links & 0.0047 & 0.02 \\
$H_{0}^{4}$ Incorrect links & 0.39 & 0.14 \\
$H_{0}^{5}$ Precision & 0.39 & 0.62 \\
$H_{0}^{6}$ Accuracy & 0.15 & 0.72 \\
\hline
\end{tabular}

For the number of incorrectly assigned links $\left(N_{i l}\right)$ (related to research question Q1), the t-test resulted in a p-value of 0.14 , so the hypothesis $H_{0}^{4}$ cannot be rejected. Furthermore, for the hypothesis $H_{0}^{5}$ (related to research question Q1) the t-test gave the p-value 0.62 and for the hypothesis $H_{0}^{6}$ (related to research question Q1) the t-test resulted in the p-value 0.72. To summarize, research question Q1 can be answered as "yes", for some aspects. Our results confirm the results from the original experiment for correctness and number of missed links but we can't confirm the result for the efficiency. As in the original experimen the hypotheses $H_{0}^{4}, H_{0}^{5}, H_{0}^{6}$ could not be rejected here. Compared to the original experime this experiment confirms no statistical difference in the number of incorrect links, orec, ion and accuracy between the two analyzed treatments. The question regarding differ $\mathrm{res}$ its for $H_{0}^{1}$ is discussed in Section 7. The summary of the original and the replica ed en ents is depicted in Table 6.

\section{Experiment results interpretation and discussion}

This section presents an interpretation of the results presented in Se tion 6. Since this experiment was conducted on a set of students, it is importa to emphasize here that the results from this study are interpreted in the light of the phere the experiment was held (Kitchenham et al 2002). The section discusses th resu s of this experiment in isolation as well as in relation to the results of the origin

\subsection{Interpretation of this experiment}

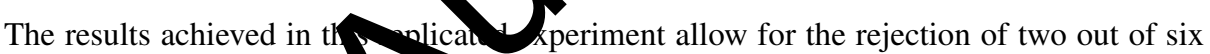
stated null hypotheses (see Tab As already mentioned, four data points were removed from the analysis for variou reasons, as described in Section 6. The results achieved by group A7 show that the subj ts were not motivated to do the task or misunderstood the task (they analyze only 3 requirements in 29 minutes). It is surprising since both subjects in this pair had one or more years of industrial experience (pairs with similar experience (A3 and M4) performed significantly better on this task), but no experience in reviewing requirements. It is an open question how they could influence the results if properly motivated. Similarly, due to unexpected tool issues (the results were not saved in the tool) we can only assume that the results achieved by groups A8 and M11 could positively influence the results for both assisted and manual method (group A8 achieved efficiency of 0.68 requirement per minute, group M11 0.4 requirement per minute and group M10 0.35 requirements 
per minute). Adding these three incomplete data points (A8, M10 and M11) to the analysis of the performance will not change the result of the hypothesis $H_{0}^{1}$ testing (although it can minimize the $\mathrm{p}$-value to 0.0890 ).

As for the $H_{0}^{1}$ (performance) hypothesis (research question Q1a), the lack of a statistically significant difference can be interpreted in the following way: we have not found this property (namely lower efficiency of the manual method comparing to the assisted method) on a different requirements management tool, namely Telelogic DOORS. The lack of statistical significance can be explained by a rather large variation in the assisted method (the minimum value for the performance is 0.29 requirement per minute while the maximum value is 0.89 requirement per minute). Furthermore, albeit the medians are almost identical for both the assisted and the manual method with respect to the performance, the range of the third quartile is much larger in the assisted method. This fact can be interpreted in favor of practical significance (Kitchenham et al 2002) in the following way: if we assume that both groups assigned to the methods are rather homogeneous, we can also assume that in both groups there are similar numbers of more and less motivated subjects. In the light of the fact that motivation has been reported to be an important determinant of productivity and quality of work in many industries (Baddoo et al 2006), the practical significance of the results is that the assisted method gives the possibility to achieve higher values of th performance than the manual method. As more motivated subjects usually achieve better results with a given task, we can assume that the top scores for both methods correspon to the most motivated pairs of subjects. The evidence reported by Badoo et al.(20 6), a eit studied on developers rather than requirements engineers, confirms that the tradith otivators of software developers, e.g. intrinsic factors, but also opportunity or ach evo ent, technically challenging work and recognition have a strong influencen ve droper's performance. Thus, when comparing the top score of both methods, v couk conclude that the assisted method may boost the performance of the motivated sub, motivated subjects.

The analysis of the results for efficiency versus the rpe forects revealed that the subjects with experience reviewing requiremen (pairs A3 and A11) were not the fastest (the values were lower or around the media value). We can postulate here that the industrial experience led these pairs to be mor when analyzing requirements. On the other hand, the top score in this group (A) was achieved by a pair of subjects that reported no industrial experience and noxp the course from which the requirements originated. Surprisingly, the twe lo st va of performance were achieved by the pairs having either one year of indust exp rience, including experience with reviewing requirements (pair A3), or experienc fron course from which the requirements originated (pair A4). In the light of se fa we can not draw any strong conclusions about the effect of both industr experience with reviewing requirements on the performance of subjects. $\mathrm{H}$ we tr, ex results show indications of negative impact of experience on the performance o he subjects. The full analysis of results of experienced versus inexperienced subjects is pres nted later in this section and in Tables 7 and 8 .

The results concerning the number of correct links (research question Q1b) can be interpreted as follows. The group that used the assisted method assigned on average $58 \%$ of the correct links, while the group that used the manual method assigned on average $43 \%$ of the correct links. The results of the t-test allows us to reject $H_{0}^{2}$. This fact may be interpreted in the following way in favor of the assisted method: even if the assisted method is put next to a rather sophisticated requirements management tool, it can still provide better support for assessing more correct links between requirements. The fact that both in the original and the replicated studies the assisted method provided a better support in linking similar 
requirements may lead to the following two interpretations: (1) the method is better in this matter, and (2) working in pairs has a minimum or equal impact on the two methods when it comes to the number of correctly linked requirements.

The results for the number of missed requirements links (research question Q1c) confirm the results of the original experiment. The t-test confirms that the assisted method can help to miss fewer requirements links than the manual method. Missing fewer links may be important when large sets of requirements have to be analyzed, which is a reasonable practical interpretation of this result. This result also confirms the interpretation that in the case of the assisted method, showing a list of similar requirements candidates limits the solution space for the analyst which results in a smaller number of missed requirements links.

Similarly to the original experiment, the results from the experiment can also not reject hypotheses $H_{0}^{4}, H_{0}^{5}$ and $H_{0}^{6}$ (research question Q1). The lack of statistically significant differences in these cases may be interpreted as the possible existence of additional factors that affect the consolidation of requirements process which were not controlled in the experiment. For example, since it is much easier to make a link in ReqSimile than in Telelogic DOORS this may affect the number of incorrect links, precision and accuracy. This threat to construct validity is described in Section 4.9 and is considered as one of the topics for further work.

The fact that subjects worked in pairs may also influence the results. Even though working in pairs has generally been considered having a positive impact on the task, for exampin in pair programming (Begel and Nachiappan 2008), the results among researcb s aro in-
consistent (Hulkko and Abrahamsson 2005, Parrish et al 2004). Therefore,asse ing he impact of working in pairs in more decision-oriented software engineeri $g$ tas $1 \mathrm{~s}$ ven more difficult. Thus, it can be assumed that working in pairs may somenting inf ence the performance of these types of tasks positively, and sometimes negat ely. this case, we assume that subjects were similarly affected by this phenomenon both the assisted and in the manual method.

The influence on the results of fluency in reading an equirements in the English language can be interpreted in the following way. Gince subir ts reported either "very good knowledge" or "'fluent knowledge' in reading a writing English our interpretation of this fact is that this aspect equally influenced al lowed to ask questions for clarification, including nders anding the requirements during the experiment. However, it remains an ope gy be the results of the experiment when performed on native English lanqua speang subjects.

The analysis of the influence of tk. ndu rial experience of the subjects of the results achieved is depicted in Table 7. That been analyzed for all pairs of subjects, as

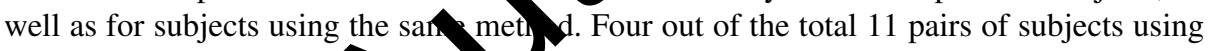
the assisted method repo havin me industrial experience. For the manual method, 5 out of 9 pairs of subject in the data analysis reported having some industrial experience. Subjects were $\mathrm{p}$ red in a way that minimizes the difference in the experience of pair members. Moreover, nly in two cases (pairs M5 and M6) were pairs formed of one experienced and one inexperienced subject (see section 4.2 for more detail about the subjects).

The analysis of the relationship of industrial experience to the results is based on the arithmetic average values of the results achieved. Table 7 shows that in most cases industrial experience negatively influenced the results (and tested hypotheses). The cells colored gray in Table 7 indicate cases where industrial experience has a positive effect on the analyzed aspect. For all hypotheses for subjects using the manual method, the industrial experience had a negative impact on the results achieved. In the case of the assisted method, the experi- 
Table 7 The analysis of the industrial experience of the subjects in relation to their results. The cells colored gray indicate cases where industrial experience had positive effect on the analyzed aspect.

\begin{tabular}{|l|l|l|l|l|l|l|}
\hline \multirow{2}{*}{} & \multicolumn{2}{|c|}{ All data } & \multicolumn{2}{c|}{ Assisted method } & \multicolumn{2}{c|}{ Manual method } \\
\cline { 2 - 7 } & Exp. & Unexp. & Exp. & Unexp. & Exp. & Unexp. \\
\hline Efficiency $H_{0}^{1}[\mathbf{N} / \mathbf{T}]$ & 0.44 & 0.48 & 0.51 & 0.52 & 0.40 & 0.42 \\
\cline { 2 - 7 } Correct $H_{0}^{2}[\%]$ & 44 & 55 & 53 & 61 & 39 & 47 \\
Missed $H_{0}^{3}[\%]$ & 55 & 44 & 46 & 38 & 60 & 52 \\
Incorrect $H_{0}^{4}[\%]$ & 39 & 54 & 40 & 66 & 38 & 33 \\
Precision $H_{0}^{5}[\%]$ & 45 & 43 & 50 & 41 & 42 & 46 \\
Accuracy $H_{0}^{6}[\%]$ & 46 & 43 & 49 & 40 & 44 & 48 \\
\hline
\end{tabular}

enced subjects made fewer incorrect links and had better precision and accuracy. The results from comparing all subjects show the same pattern as the results for the assisted method. While the results are implausible, they may be an indicator that general software engineering industrial experience may not be useful in the task of analyzing requirements, at least when the experience is minimal to small. Thus, we state a hypothesis that experience in reviewing requirements and the domain knowledge should significantly help in achieving better results by our subject. Keeping in mind the scarceness of the data, we provide some examples th we used to support the hypothesis in the text that follows.

Six pairs of subjects reported having experience analyzing and reviewing requiraments outside of the courses taken in their education. In one case (M8), a person with fore an one year of industrial experience was paired with a person with no experience. tho gh it may be expected that an experienced pair member can significantly inflence e rults of this pair, the efficiency of this pair was only 0.02 higher than the mal va of the efficiency for the subjects using the manual method. The number of corrent links is $10 \%$ lower, the number of incorrect links is $10 \%$ higher while precision a lac aracy are very close to the average values for the entire group using the mal method. In the case of pair M7, a person with experience only from courses ais aith a person with less than a year of industrial experience in analyzing and r rewing quirements. The results achieved by this pair are higher than the average in ters of efficiency ( $7 \%$ higher), close to the average for the share of correct links, and belo ane for the remaining attributes (30\% more incorrect links than the average, 16\% lowe than the average for the precision and $12 \%$ lower than the average for the acuining 3 pairs of subjects (A1 and M5) were composed of a person with anl aden experience with a person with less than a year of industrial experience. Pair M naly 0.4 requirement per minute which is close to the average value $(0.41)$, achieved $\%$ correct links (the average value is $43 \%$ for this group) and $44 \%$ of incorrect link The ecision achieved by pair M5 is 50\% which is $4 \%$ higher than the average. $W$ it con accuracy, pair M5 achieved $46 \%$ accuracy (the average value was 46\%). T e ror for efficiency for pairs (A1, A3 and A11) were below or about the average values and these data points could have been partly responsible for the fact that hypothesis $H_{0}^{1} \mathrm{cold}$ not be rejected. The results for these pairs for the number of correct links and the share of missed links were also below the average and the median values. The results for the number of incorrect links were around the mean value and above the median value. Finally, the results for the precision and accuracy were below the median values. To summarize, the influence of experience in analyzing and reviewing requirements can't be clearly defined and statistically confirmed, as subjects report both results above and below the average values.

As the last step of the analysis, we investigate whether prior experience in the course that originated the requirements in some manner influences the results achieved by the sub- 
Table 8 The analysis of the experience from the course where requirements originate from. $E$ denoted that both pair members are experienced, $E$ and $U$ denoted one experienced and inexperienced person working together and $U$ denoted that both pair members were inexperienced.

\begin{tabular}{|l|l|l|l|l|l|l|l|l|l|}
\hline & \multicolumn{3}{|c|}{ All data } & \multicolumn{3}{c|}{ Assisted method } & \multicolumn{3}{c|}{ Manual method } \\
\hline & $\mathrm{E}$ & $\mathrm{E}$ and U & $\mathrm{U}$ & $\mathrm{E}$ & $\mathrm{E}$ and U & $\mathrm{U}$ & $\mathrm{E}$ & $\mathrm{E}$ and U & $\mathrm{U}$ \\
\hline Efficiency $H_{0}^{1}[\mathbf{N} / \mathbf{T}]$ & 0.45 & 0.39 & 0.57 & 0.53 & 0.38 & 0.69 & 0.37 & 0.40 & 0.46 \\
\cline { 4 - 11 } Correct $H_{0}^{2}[\%]$ & 56 & 51 & 46 & 68 & 53 & 58 & 46 & 48 & 34 \\
Missed $H_{0}^{3}[\%]$ & 50 & 57 & 34 & 56 & 59 & 31 & 44 & 55 & 37 \\
\cline { 2 - 11 } Incorrect $H_{0}^{4}[\%]$ & 58 & 54 & 30 & 85 & 58 & 56 & 31 & 50 & 27 \\
\cline { 2 - 11 } Precision $H_{0}^{5}[\%]$ & 42 & 39 & 50 & 40 & 37 & 56 & 45 & 42 & 44 \\
Accuracy $H_{0}^{6}[\%]$ & 43 & 41 & 49 & 39 & 39 & 53 & 47 & 45 & 46 \\
\hline
\end{tabular}

jects. We can assume that this experience can somehow be compared to specific domain knowledge. In the course model, all team members are actively involved in analyzing and reviewing requirements. Thus, we only distinguish between subjects that took and did not take the course. For six pairs of subjects, both subjects have experience from the course, for seven other pairs only one pair member had experience from the course. Finally for six pairs, both subjects reported no experience from the course. The pairs where both member had experience and where both members had no experience were equally distributed between the methods (three pairs for each method). The assisted method had four pairs wh experience and lack of experience. The analysis is depicted in Table 8. Pairs where ooth air members had experience from the course are abbreviated with the letter "E", wher nnly ne pair member had experience are abbreviated with "E and U" and where non of th th pair members had any experienced from the course is abbreviated with the lette "U".

Analyzing the differences between the average results for all thre sub-aroups for both methods we can see that the expected behavior can only be confirmed the humber of correct links. Pairs with experience in the course achieved better cowectness than inexperienced pairs, independent of whether one or both members had he pee Another interesting observation here is that inexperienced subjects missed on average 9 y $34 \%$ of requirements links, while experienced subjects respectively missed $5 \%$ (both pair members experienced) and $57 \%$ (when one of the pair members had som nee). The lowest average precision and accuracy levels were recorded for pair wher one pair member had experience from the course from which the requirement 1 . The analysis of the pairs working with the same method confirms the apaly of a data points. For the manual method experienced pairs turned out to be more rrec han the inexperienced pairs. For the assisted method, pairs where both members hero verienced were more correct, but pairs where only one member had experienc when not as correct as the inexperienced pairs. Finally, the pairs where only one perienced performed worse in all aspects for both methods analyzed than the air pre both persons were experienced.

\subsection{Interpretation of the results from both experiments}

In this section, we provide the analysis and discussion of the results achieved in both the original and the replicated experiments. We have used standard t-tests to test if there are any significant differences between the two experiments. From the results of the t-tests between the same methods depicted in Table 9, we can see no significant difference for any of the cases (research question Q2). However, some interesting differences between the two experiments for the efficiency of the subjects using the assisted method can be seen from the 
Table 9 The results of the t-tests for the original and the replicated experiments for the same methods.

\begin{tabular}{lll}
\hline Hypotheses & $\begin{array}{l}\text { Assisted old/new (p-value) } \\
\text { (research question Q2a) }\end{array}$ & $\begin{array}{l}\text { Manual old/new (p-value) } \\
\text { (research question Q2b) }\end{array}$ \\
\hline$H_{0}^{1}$ Efficiency & 0.48 & 0.27 \\
$H_{0}^{2}$ Correct links & 0.93 & 0.30 \\
$H_{0}^{3}$ Missed links & 0.37 & 0.20 \\
$H_{0}^{4}$ Incorrect links & 0.21 & 0.73 \\
$H_{0}^{5}$ Precision & 0.81 & 0.45 \\
$H_{0}^{6}$ Accuracy & 0.90 & 0.41 \\
\hline
\end{tabular}

box-plot visualization in Fig. 7. As can be seen in Fig. 7, the results for the efficiency of the assisted method in this experiment have a much larger range of values, which may be the reason why the hypothesis $H_{0}^{1}$ could not be rejected (research question Q2a). As described in Section 7.1 the two lowest values of performance were achieved by the pairs having either one year of industrial experience, including experience from reviewing requirements (pair A3), or experience from the course from which the requirements originate (pair A4).

However, one of the possible explanations for the difference between the performance in this experiment and in the original experiment differs may be that more advancedmarch ing and filtering functionalities have been used in the current manual method. the original experiment, the manual method in this experiment uses advanced anard ing nd filtering functionalities which may (to some extent) be comparable to the xical im. rity analysis because they also present only a subset of analyzed requiremen and the analyst using the filtering and searching functionality has to provide a me ningful search string to filter out similar requirements, while in the lexical similarity the analysis is done automatically. Our interpretation is supported by Fig. 8 y depicts the results of the performance for the manual method between the origh a d eplicated experiments. The median value for this replication study is 0.41 and 0.05 her than in the original experiment (0.36). However, the second quartile has ne diverse values than in the original experiment. Moreover, we can assume that th method has a higher degree of uncertainty which is shown by the results for the ccura $y$.

\subsection{Discussion}

In this section, we discus signs for this experiment as well as the differences between the two experime se seplication has been run in two sesiong alternative design could be neficial for the comparative analysis of the original and the replicated experiment (RQ2). Vor example the paired t-test could have been used to support comparison between this replication study and the original study (Kachigan 1991). However, the it remains an open question if paired units are similar with respect to "noise factors" for both the assisted and the manual methods used. It could also have been beneficial to the validity if the subjects answered the pre-questionnaire before running the study and were then assigned to treatments based on the result of this questionnaire. The manual analysis of the two experiment sessions did not reveal any significant differences between the Tuesday and the Friday sessions. However, to fully address this threat to validity, additional statistical tests should be used. Finally, changing the design of the study to use random samples of 30 
The results of the efficiency of the assisted method in the original and the replicated experiments

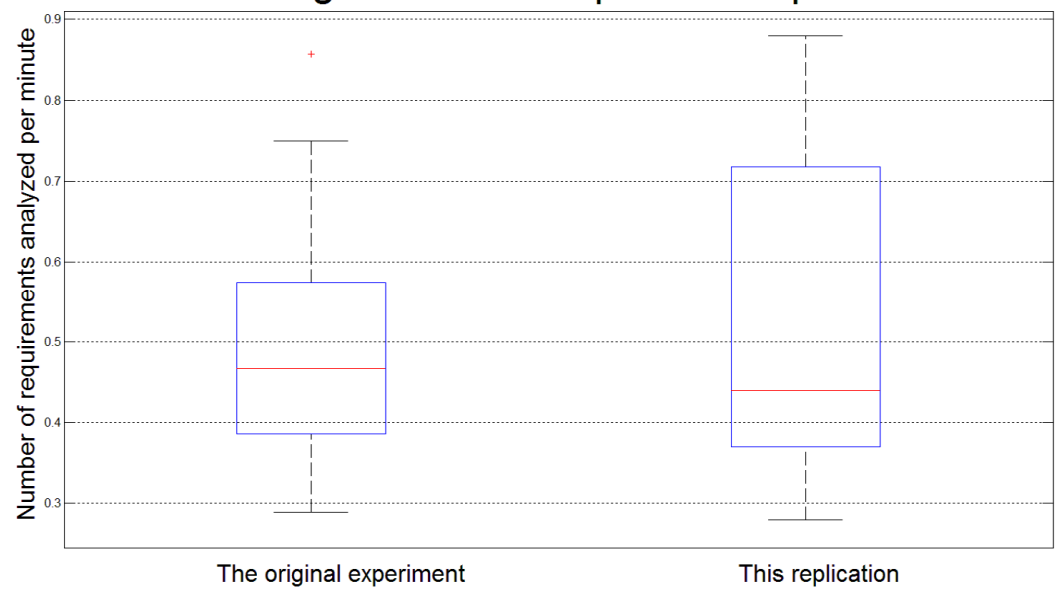

Fig. 7 The result of comparing the efficiency of the assisted method in two experiment sessions.

The results of the efficiency of the manual methoa in the original and the replicated experime its

Fig. 8 The results of the efficienc, achieved by the manual method in the original and the replicated experiments.

and 160 requirements for each subject, generated from a much large dataset of requirements is one of the options for further work.

During this experiment six hypotheses were tested using the same data set, and more tests were performed comparing the data from the original and replicated experiments. The result of performing multiple comparisons on the same data is increased probability of Type I error, which in case of only one comparison is equal to the obtained p-value (Arcuri and 
Briand 2011). Thus, the Bonferroni correction should be discussed here. In this case, we performed 18 tests, 6 tests comparing the assisted and manual method (to answer the research question Q1), 6 tests comparing the old/new experiments with regard the assisted method (to answer the research question Q2a) and 6 tests comparing the old/new experiments with regard to the manual method (to answer the research question $\mathrm{Q} 2 \mathrm{~b}$ ). This yields a significance level of $0.05 / 18=0.0027$ according to Bonferroni. In this case it is no longer possible to reject hypotheses $H_{0}^{2}$ and $H_{0}^{3}$. The correction has no impact on the results of the tests between the original and the replicated experiments. However, the correction has not been used in the original experiment and has been criticized by a number of authors (Arcuri and Briand 2011, Perneger 1998, Nakagawa 2004) where some of them do not recommend using the Bonferroni adjustment (Arcuri and Briand 2011). In the light of this criticism, it is an open question for this work as to whether or not this correction should be used. Therefore, we report the obtained $\mathrm{p}$-values for all performed tests in case the readers want to evaluate the results using the Bonferroni correction or other adjustment techniques (Arcuri and Briand 2011).

The relationship between the efficiency and the practical experience of the subjects may have been investigated using multivariate analysis. For example, understanding if the efficiency of the subjects was related to their accuracy could have been investigated by recordin the efficiency of linking requirements at random as a reference point. Since this has not been done, we consider this analysis as possible future work and thus outside the scope of thr article.

\section{Conclusions}

Large market-driven software companies face new challenges that eme du to their extensive growth. Among those challenges, a need for efficient methods to anayze large numbers of requirements, issued by various customers and other take old has emerged (Regnell and Brinkkemper 2005). The result is an increasing effondearcate to analyzing incoming requirements against those requirements already analy d or implemented. This task is also called requirements consolidation. The core of the-equents consolidation process is finding the similarities between requirements and recor ing them by making links between them (Natt och Dag et al 2006).

In this paper, we present a replicated erim that aims to assess whether a linguistic method supports the requirements cor liak ion task better than a searching and filtering method. In this experiment, two metk ds plemented in two different tools were compared for the requirements consolidation tas The assisted method, which utilizes natural language processing algorith to pro ide similarity list for each analyzed requirements, was compared with the manua, mey which utilizes searching and filtering algorithms to find similar requirements. After clding which requirements were similar, the subjects assigned links between the requiremen. The conclusions of this paper are as follows:

- Subjects using the assisted method were statistically not more efficient in consolidating requirements than the subjects using the manual method (research question Q1a), which is a different result compared to the original study

- The assisted method was confirmed as significantly more correct in consolidating requirements than the manual method (the manual method was changed from the original experiment) (research question Q1b), which is inline with the original study. 
- The assisted method helps to miss fewer requirements links than the manual method (research question Q1c), which is the same result as in the original study.

- The hypotheses that could not be rejected in the original study (in terms of the number of incorrect links, precision and accuracy related to research question Q1) could also not be rejected in this experiment. Further investigation is required to understand the reasons for these results.

- The analysis of the results achieved for the same method (assisted or manual) between the original and the replicated study (research question Q2) shows no significant difference in any of the cases. However, some differences in favor of the searching and filtering method have been observed between the results of the performance of the subjects using the manual methods.

To summarize, for two of our hypotheses the results reported in this replication study confirm the results achieved in the original experiment. The first confirmed hypothesis $\left(H_{0}^{2}\right.$ is that the assisted method helps to make more correct links than the manual method The second confirmed hypothesis $\left(H_{0}^{3}\right)$ indicates that the assisted method helps to miss fewern quirements links than the manual method. The statistical significance in performa ce or the assisted method achieved over the manual method (hypothesis $H_{0}^{1}$ ) is not confirm $\mathrm{d}$ in his study. The remaining three hypotheses regarding the number of incorrect $1 \mathrm{ks}$ ( $\mathrm{pa}$ esis $H_{0}^{4}$ ), precision (hypothesis $H_{0}^{5}$ ) and accuracy (hypothesis $H_{0}^{6}$ ) could nothe eject d, which is the same situation as reported in the original experiment (Natt oc Dag et 2006). In order to investigate the possible reasons for the difference in the rema ing ase, this paper provides a cross-case analysis of the same methods across the wo experiment sessions as well as detailed analysis of the relations between the subjects and their results.

The analysis revealed that the pairs of subjects with xperience in the course that originated the requirements achieved better correctnes tha, ne.perienced pairs, independent of whether one of both members had the experiend At $t$ e same time, the pairs of subjects without any experience missed on avera ex requirements links than the experienced pairs of subjects. Furthermore, the par on one person had experience performed worse in all aspects than the pairs wher bothersons were experienced. The analysis revealed no statistical difference for an of the cases (which refers to the research question RQ2). However, the analysis of th effil ency of the subjects using the assisted method in the two experiments, depi tea Fig., revealed that the values for the efficiency achieved in this replication have a nigner range. The results for the efficiency of the manual methods in the two experime ts, depicted in Fig. 8, shows similar range of values, but different medians. It should be moted that there are validity threats to the study as described in Section 4.9, e. g. experience of the subjects and their incentives, the number of subjects participating, requirements used in the experiment, and the multiple comparison threats.

Performing a third experiment with experienced practitioners and requirements sets from industry is left for future work. Moreover, it would be interesting to further investigate the influence of working in pairs on the requirements consolidation task as well as to analyze the influence of the construction of the user interfaces on the efficiency of correctness of the subjects. 
Acknowledgements This work is supported by VINNOVA (Swedish Agency for Innovation Systems) within the UPITER project. Special acknowledgments to Richard Berntsson-Svensson for participating in the pilot study and reviewing the paper. We are also thankful to Lars Nilsson and David Callele for reviewing the paper and excellent language comments.

\section{References}

Aguilera C, Berry D (1991) The use of a repeated phrase finder in requirements extraction. J Syst Softw 13:209-230, DOI 10.1016/0164-1212(90)90097-6

Antoniol G, Canfora G, Casazza G, De Lucia A, Merlo E (2002) Recovering traceability links between code and documentation. Software Engineering, IEEE Transactions on 28(10):970 - 983, DOI 10.1109/TSE.2002.1041053

Arcuri A, Briand L (2011) A practical guide for using statistical tests to assess randomized algorithms in software engineering. In: Proceeding of the 33rd international conference on Software engineering, ACM, New York, NY, USA, ICSE '11, pp 1-10, DOI 10.1145/ 1985793.1985795, URL http://doi .acm.org/10.1145/1985793.1985795

Baddoo N, Hall T, Jagielska D (2006) Software developer motivation in a high maturity company: a case study. Software Process: Improvement and Practice" pp 219-228

Basili V, Shull F, Lanubile F (1999) Building knowledge through families of experiments. Software Engineering, IEEE Transactions on 25(4):456 -473, DOI 10.1109/32.7009

Begel A, Nachiappan N (2008) Pair programming: what's in it for me? In: ESEM 08: ceedings of the Second ACM-IEEE international symposium on Empirical aftm gineering and measurement, ACM, New York, USA, pp 120-128, DOI 1( 1145, 4 - 04. 1414026

Berenbach B, Paulish DJ, Kazmeier J, Rudorfer A (2009) Software \& Sy tems Requirements Engineering: In Practice. Pearson Education Inc.

Breaux TD (2009) Exercising due diligence in legal requiments acquisition: A toolsupported, frame-based approach. Atlanta, GA, UA tapp 225 - 230, DOI 10.1109/RE.2009.46

Cleland-Huang J, Chang CK, Ge Y (2002) Supporting \& nnt based traceability through highlevel recognition of change events. In: COMPS 10 2. Proceedings of the 26th International Computer Software and Applications onfer ice on Prolonging Software Life: Development and Redevelopment, IFEE aposiety, Washington, DC, USA, pp 595-602

Cleland-Huang J, Settimi R, Duan C, $\mathrm{C}$ (2005) Utilizing supporting evidence to improve dynamic requirements traced ility : Proceedings of the 13th IEEE International Conference on Requirements gined (RE 2005), pp 135-144

Cleland-Huang J, Berenb C Cla Settimi R, Romanova E (2007) Best practices for automated traceability. Om atw 40(6):27-35, DOI 10.1109/MC.2007.195

Cleland-Huang J, Czaudern A, Gibiec M, Emenecker J (2010) A machine learning approach for tracing regulato codes to product specific requirements. In: ICSE '10: Proceedings of the 32nd ACM/IEEE International Conference on Software Engineering, ACM, New York, NY, USA, pp 155-164, DOI 10.1145/1806799.1806825

ECTS (2010) The ECTS grading systems defined by European Commission. http://en . wikipedia.org/wiki/ECTS\_grading \_scale, accessed 07/09/2011

Fabbrini F, Fusani M, Gnesi S, Lami G (2001) An automatic quality evaluation for natural language requirements. In: Proceedings of the 7th International Workshop on Requirements Engineering Foundation for Software Quality (REFSQ 2001), pp 4-5, DOI 10.1.1.9.7525 
Fantechi A, Gnesi S, Lami G, Maccari A (2003) Applications of linguistic techniques for use case analysis. Requir Eng 8(3):161-170, DOI 10.1007/s00766-003-0174-0

Fricker S, Gorschek T, Byman C, Schmidle A (2010) Handshaking with implementation proposals: Negotiating requirements understanding. IEEE Software 27:72-80, DOI 10. 1109/MS.2009.195

Gacitua R, Sawyer P, Gervasi V (2010) On the effectiveness of abstraction identification in requirements engineering. In: Requirements Engineering Conference (RE), 2010 18th IEEE International, pp 5 -14, DOI 10.1109/RE.2010.12

Gervasi V (1999) Environment support for requirements writing and analysis. $\mathrm{PhD}$ thesis, University of Pisa

Gervasi V, Nuseibeh B (2000) Lightweight validation of natural language requirements: A case study. In: Proceedings Fourth International Conference on Requirements Engineering. ICRE 2000, IEEE Comput. Soc, pp 113-133, DOI 10.1.1.28.9876

Goldin L, Berry DM (1997) Abstfinder, a prototype natural language text abstraction finder for use in requirements elicitation. Autom Softw Eng 4:375-412, DOI 10.1.1.26.8152

Gorschek T, Garre P, Larsson SBM, Wohlin C (2007) Industry evaluation of the requirements traction model. Requirements Engineering 12(3):163-190, DOI 10.1007/ s00766-007-0047-z

Gotel O, Finkelstein C (1994) An analysis of the requirements traceability problem In. Requirements Engineering, 1994., Proceedings of the First International Conference on pp 94 -101, DOI 10.1109/ICRE.1994.292398

Hayes J, Dekhtyar A, Osborne J (2003) Improving requirements tracing via in cma on retrieval. In: Requirements Eng Conf, 2003. Proc. 11th IEEE Int, pp $18-7$, 8 OI 10.1109/ICRE.2003.1232745

Hayes J, Dekhtyar A, Sundaram S, Holbrook E, Vadlamudi S, Ap A (2007) Requirements tracing on target (retro): improving software maintenance hro gh traceability recovery. Innovations in Systems and Software Engineerip 3:193-202, DOI 10.1007/ s11334-007-0024-1, 10.1007/s11334-007-0024-1

Hayes JH, Dekhtyar A, Sundaram SK (2006) Advancip candida link generation for requirements tracing: The study of methods. IEEE ans Softw Eng 32(1):4-19, DOI 10.1109/TSE.2006.3

Higgins S, Laat M, Gieles P, Geurts E (2003) M nagin requirements for medical it products. IEEE Software 20(1):26-33

Höst M, Wohlin C, Thelin T (2005) Expe henta context classification: Incentives and experience of subjects. In: Proceeding of th 27:th International Conference on Software Engineering (ICSE), pp 470-478

Hulkko H, Abrahamsson P (2005 A mu ple case study on the impact of pair programming on product quality. In: Software Engineering, A CM York, NY, USA, pp 495-504, DOI 10.1145/1062455. 1062545

IBM (2010a) Rational doors (brmer telelogic doors) product description. http: //www-01 . ibm.com/software/awdtools/doors/productline/, Accessed 07/09/2011

IBM (2010b) Rational doors product description (former telelogic doors), accessed 15.04.2010. http://www-01.ibm.com/software/awdtools/doors/productline/, Accessed 07/09/2011

IEEE (2010) The ieee keyword taxonomy webpage. http://www.computer.org/mc/ keywords/software.htm, Accessed 07/09/2011

Ivarsson M, Gorschek T (2009) Technology transfer decision support in requirements engineering research: a systematic review of REj. Requir Eng 14(3):155-175, DOI 
10.1007/s00766-009-0080-1

Jackson P, Moulinier I (2002) Natural language processing for online applications. Text retrieval, extraction and categorization, Natural Language Processing, vol 5. Benjamins, Amsterdam, Philadelphia

Jarke M (1998) Requirements tracing. Commun ACM 41:32-36, DOI 10.1145/290133. 290145

Kachigan SK (1991) Multivariate statistical analysis: A conceptual introduction. Radius Press

Kamsties E, Berry DM, Paech B (2001) Detecting ambiguities in requirements documents using inspections. In: Proceedings of the First Workshop on Inspection in Software Engineering (WISE 2001), pp 68-80, DOI 10.1.1.93.4870

Karlsson L, sa G Dahlstedt A, Natt Och Dag J, Regnell B, Persson A (2002) Challenges in market-driven requirements engineering - an industrial interview study. In: Proceedings of the Eighth International Workshop on Requirements Engineering: Foundation for Software Quality (REFSQ 2002)

Kitchenham B, Pfleeger SL, Pickard LM, Jones PW, Hoaglin DC, Emam E, Rosenberg J (2002) Preliminary guidelines for empirical research in software engineering. IEEE Trans Softw Eng 28(8):721-734, DOI 10.1109/TSE.2002.1027796

Konrad S, Gall M (2008) Requirements engineering in the development of large-scale systems. In: Proceedings of the 16th International Requirements Engineering Conferents (RE 2008), pp 217-222

Kotonya G, Sommerville I (1998) Requirements Engineering. John Wiley \& Sons

Lauesen S (2002) Software Requirements - Styles and Techniques. Addiso -We
Leuser J (2009) Challenges for semi-automatic trace recovery in the anto domain. In: TEFSE '09: Proceedings of the 2009 ICSE Workshop on Tra eabilit in Emerging Forms of Software Engineering, IEEE Computer Society, Washingtd D , USA, pp 3135, DOI 10.1109/TEFSE.2009.5069580

Lin J, Lin CC, Huang J, Settimi R, Amaya J, Bedford C Be ent B, Khadra O, Duan C, Zou X (2006) Poirot: A distributed tool supporting enterprise-wid automated traceability. In: Requirements Engineering, 14th IEEE Internati al Conference, pp 363 -364, DOI 10.1109/RE.2006.48

Lormans M, Van Deursen A (2006) Can lsi help econs ucting requirements traceability in design and test? In: CSMR '06: Proceedip onference on Software Maintenance and Reengineering, IEEE Compute So ty, hington, DC, USA, pp 47-56

Lucia AD, Fasano F, Oliveto R, Torto G (207) Recovering traceability links in software artifact management systems using nfo, ron retrieval methods. ACM Trans Softw Eng Methodol 16(4):13, DOI $10.1 \times 5 / 12,933.1276934$

Lund University (2011a) requin $m$ th engineering course (ets 170) at the lund university. http://www.cs.1t .s \$170/, Accessed 07/09/2011

Lund University (2011b) Th software development of large systems course page at lund university. http://cs.1th_se/etsn05/, Accessed 07/09/2011

Macias B, Pulman SG (1995) A method for controlling the production of specifications in natural language. Comput J 48(4):310-318

Manning CD, Schütze H (2002) Foundations of Statistical Natural Language Processing. MIT Press

Marcus A, Maletic JI (2003) Recovering documentation-to-source-code traceability links using latent semantic indexing. In: ICSE '03: Proceedings of the 25th International Conference on Software Engineering, IEEE Computer Society, Washington, DC, USA, pp $125-135$ 
Mich L, Mylopoulos J, Nicola Z (2002) Improving the quality of conceptual models with nlp tools: An experiment. Tech. rep., University of Trento, DOI 10.1.1.62.6397

Nakagawa S (2004) A farewell to bonferroni: the problems of low statistical power and publication bias. Behavioral Ecology 15(6):1044-1045, DOI 10.1093/beheco/arh107, URL http://beheco.oxfordjournals.org/content/15/6/1044. short, http://beheco. oxfordjournals.org/content/15/6/1044.full.pdf+html

Natt och Dag J (2006a) Managing natural language requirements in large-scale software development. PhD thesis, Lund University, Sweden

Natt och Dag J (2006b) The reqsimile tool website. http://reqsimile.sourceforge. net/, Accessed 07/09/2011

Natt och Dag J, Gervasi V, Brinkkemper S, Regnell B (2004) Speeding up requirements management in a product software company: Linking customer wishes to product requirements through linguistic engineering. In: Proceedings of the 12th International Requirements Engineering Conference (RE 2004), pp 283-294

Natt och Dag J, Thelin T, Regnell B (2006) An experiment on linguistic tool support for consolidation of requirements from multiple sources in market-driven product development. Empirical Software Engineering 11(2):303-329, DOI 10.1007/s10664-006-6405-5

Panis MC (2010) Sucessful deployment of requirements traceability in a commercial eng neering organization ... really. In: Proceedings of the 18th IEEE International Reguirements Engineering Conference, pp 303-307

Parrish A, Smith R, Hale D, Hale J (2004) A field study of developer pairs: Pr duct ity impacts and implications. IEEE Softw 21(5):76-79, DOI 10.1109/MS.2004-13 306

Perneger TV (1998) What's wrong with Bonferroni adjustments, vol 316

Pohl K, Bockle G, van der Linden FJ (2005) Software Product Line Ensin ring Foundations, Principles and Techniques. Springer

Ramesh B, Jarke M (2001) Toward reference models for requiremen tra eability. IEEE Trans Softw Eng 27(1):58-93, DOI 10.1109/32.895989

Ramesh B, Powers T, Stubbs C, Edwards M (1995) Imp ne in uirements traceability: a case study. In: RE '95: Proceedings of the Second HEE Inte dational Symposium on Requirements Engineering, IEEE Computer Society, Vashington, DC, USA, p 89

Rayson P, Emmet L, Garside R, Sawyer P (2001) project: Experiments with the application of probabilistic NLP to systems eng neeril . In: Natural Language Processing and Information Systems, Springer, Lect Computer Science, vol 1959, pp 288-300, DOI 10.1007/3-540-45399-入 24

Regnell B, Brinkkemper S (2005) E ined ng and Managing Software Requirements, Springer, chap Market-Driven Req ren Engineering for Software Products, pp 287308

Regnell B, Beremark P, K ndh O 8) A market-driven requirements engineering process: Results from an in us arocess improvement programme. Requirements Engineering 3(2):121-129, DQ 10.1007/BF02919972

Rolland C, Proix C (1992) A hatural language approach for requirements engineering. In: Advanced Information Systems Engineering, Springer Berlin / Heidelberg, Lecture Notes in Computer Science, vol 593, pp 257-277, DOI 10.1007/BFb0035136

Rupp C (2000) Linguistic methods of requirements engineering (NLP). In: Proceedings of the EuroSPI 2000, pp 68-80

Ryan K (1993) The role of natural language in requirements engineering. In: Proceedings of the IEEE International Symposium on Requirements Engineering, San Diego California, IEEE Computer Society Press, pp 240-242, DOI 10.1.1.45.7180 
Samarasinghe R, Nishantha G, Shutto N (2009) Total traceability system: A sustainable approach for food traceability in smes. In: Industrial and Information Systems (ICIIS), 2009 International Conference on, pp 74 -79, DOI 10.1109/ICIINFS.2009.5429887

Sawyer P, Cosh K (2004) Supporting measur-driven analysis using nlp tools. In: Proceedings of the 10th International Workshop on Requirements Engineering: Foundations of Software Quality (REFSQ 2004), pp 137-142

Sawyer P, Rayson P, Garside R (2002) REVERE: Support for requirements synthesis from documents. Inf Syst Front 4(3):343-353, DOI 10.1023/A:1019918908208

Sawyer P, Rayson P, Cosh K (2005) Shallow knowledge as an aid to deep understanding in early phase requirements engineering. IEEE Trans Softw Eng 31(11):969-981, DOI 10.1109/TSE.2005.129

Shull FJ, Carver JC, Vegas S, Juristo N (2008) The role of replications in empirical software engineering. Empir Software Eng 13(2):211-218, DOI 10.1007/s10664-008-9060-1

Sjøberg DIK, Hannay JE, Hansen O, Karahasanovic VB, Liborg A, Rekdal NK (2005) The survey of controlled experiments in software engineering. IEEE Trans Softw Eng 31(9):733-753, DOI 10.1109/TSE.2005.97

Sommerville I, Sommerville I, Sawyer P, Sawyer P (1997) Viewpoints: principles, problems and a practical approach to requirements engineering. Ann Softw Eng 3:101-130

Strens M, Sugden R (1996) Change analysis: a step towards meeting the challenge of changing requirements. In: Engineering of Computer-Based Systems, 1996. Proceedings JEs Symposium and Workshop on, pp 278 -283, DOI 10.1109/ECBS.1996.494539

Wiegers KE (2003) Software Requirements, Second Edition. Microsoft Press_Uh ht p: //www . worldcat .org/isbn/0735618798

Wilkinson L (1999) Statistical methods in psychology journals: Guidelin and explannations. American Psychologist 54(8):594-604

Wilson W, Rosenberg LH, Hyatt L (1997) In: ICSE '97: Proceedings f th 19th international conference on Software engineering, ACM, New Y DOI 10.1145/253228.253258

Wohlin C, Runeson P, Höst M, Ohlsson MC, Regnell B, Hesslep_ 2000) Experimentation in Software Engineering An Introduction. Kluwer A demic Publishers

Zowghi D, Offen R (1997) A logical framework fo do ing and reasoning about the evolution of requirements. In: Requirements Eng heerin, 1997., Proceedings of the Third IEEE International Symposium on, p 247 5 10.1109/ISRE.1997.566875

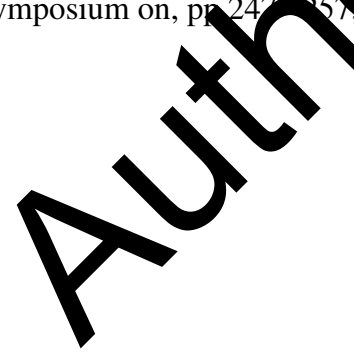

